\title{
Metric wave approach to flexoelectricity within density functional perturbation theory
}

\author{
Andrea Schiaffino, ${ }^{1}$ Cyrus E. Dreyer, ${ }^{2,3,4}$ David Vanderbilt, ${ }^{2}$ and Massimiliano Stengel ${ }^{5,1}$ \\ ${ }^{1}$ Institut de Ciència de Materials de Barcelona (ICMAB-CSIC), Campus UAB, 08193 Bellaterra, Spain \\ ${ }^{2}$ Department of Physics and Astronomy, Rutgers University, Piscataway, New Jersey 08845-0849, USA \\ ${ }^{3}$ Department of Physics and Astronomy, Stony Brook University, Stony Brook, New York 11794-3800, USA \\ ${ }^{4}$ Center for Computational Quantum Physics, Flatiron Institute, 162 th Avenue, New York, New York 10010, USA \\ ${ }^{5}$ ICREA-Institució Catalana de Recerca i Estudis Avançats, 08010 Barcelona, Spain
}

(Received 4 December 2018; published 5 February 2019)

\begin{abstract}
Within the framework of density functional perturbation theory, we implement and test a "metric wave" response-function approach. It consists in the reformulation of an acoustic phonon perturbation in the curvilinear frame that is comoving with the atoms. This means that all the perturbation effects are encoded in the first-order variation of the real-space metric, while the atomic positions remain fixed. This approach can be regarded as the generalization of the uniform strain perturbation of Hamann et al. [D. R. Hamann, X. Wu, K. M. Rabe, and D. Vanderbilt, Phys. Rev. B 71, 035117 (2005)] to the case of inhomogeneous deformations, and greatly facilitates the calculation of advanced electromechanical couplings such as the flexoelectric tensor. We demonstrate the accuracy of our approach with extensive tests on model systems and on bulk crystals of $\mathrm{Si}$ and $\mathrm{SrTiO}_{3}$.
\end{abstract}

DOI: 10.1103/PhysRevB.99.085107

\section{INTRODUCTION}

Theoretical attention to the fundamentals of mechanical deformations is of growing importance due to a surge of interest [1-3] in the flexoelectric effect, i.e., the polarization (P) response of a generic insulator to a strain gradient deformation. The renewed activity on flexoelectricity has mainly been motivated by a number of promising experimental results, demonstrating its large potential in different applications such as sensors and MEMS [4], memory storage [5], and replacement of piezoelectrics [6]. This experimental excitement motivates the urgency of supporting the results with a robust and predictive theory.

In this context, density functional theory (DFT) appears as the most natural approach to study electromechanical response properties with unbiased quantum-mechanical accuracy. Techniques for calculating piezoelectricity ( $\mathbf{P}$ response to uniform strain) are now well established [7,8]; however, generalization to flexoelectricity is far from trivial, and viable methodologies have started to appear only very recently [913]. Their conceptual basis consists in the long-wave analysis of acoustic phonon perturbations. This has the advantage of recasting a strain gradient, which breaks translational symmetry, into a periodic problem, by exploiting the standard treatment of incommensurate perturbations within the context of density functional perturbation theory (DFPT). Since we are interested in the electric response of insulators to mechanical deformations, the relevant quantity on which we have to focus our attention is the polarization response. By taking its longwave expansion, we can systematically identify the lowest orders in the wave vector $\mathbf{q}$ with specific electromechanical couplings $[10,14]$. The zeroth-order term, related to rigid translations of the lattice, vanishes due to the acoustic sum rule; the first-order term is the piezoelectric coefficient; the second-order term corresponds to the flexoelectric coefficient.
Generally speaking, the total polarization response of the crystal includes both purely electronic and lattice-mediated contributions. While the latter are relatively uncomplicated to understand and calculate, as the corresponding formulas bear many similarities to those that are valid for simplified pointcharge models [15], for the clamped-ion contribution to the polarization the knowledge of the microscopic current-density response $[9,10]$ to the deformation is needed. Despite the fundamental nature of this observable in quantum mechanics [16], the current density is not routinely available in public DFT codes. It is only recently that some of us established a computationally tractable definition of the current density and used it in the context of phonon perturbations to obtain the flexoelectric coefficients of selected materials [12]. This strategy represents a methodological breakthrough, as it allows the calculation of all of the independent components of the bulk flexoelectric tensor by using a primitive crystal cell.

There are further subtleties, however, that the approach of Ref. [12] has addressed only partially. For example, it has become clear in the past few years that flexoelectricity is not a genuine bulk property: before attempting any comparison between ab initio results and experiments, the bulk flexoelectric tensor needs to be combined with the relevant surface contributions, i.e., those coming from "surface piezoelectricity" $[10,17]$. Surface effects might appear, at first sight, irrelevant in the context of a bulk theory; yet, the separation between surface and bulk contributions to the flexoelectric response is not unique.

A manifestation of this issue was illustrated in Ref. [13], where it was shown that the electronic flexoelectric tensor consists of two distinct physical contributions: a "static" and a "dynamic" term. The latter, in particular, is related to rotation gradients (a subset of the strain-gradient tensor components), and is proportional to the orbital magnetic susceptibility tensor. While both mechanisms contribute to the physical 
current-density field that is generated by a strain gradient, and therefore are implicitly present in the bulk response as calculated via a standard phonon perturbation, only the former is relevant to the electromechanical response of a finite sample. (The rotation-gradient contribution, being a purely solenoidal current, makes no contribution to the charge density in the bulk, while its effect at the boundary is exactly canceled by an equal and opposite surface term.) Therefore, in Ref. [12] an independent calculation of the diamagnetic susceptibility was performed in order to isolate the physically relevant static part.

An alternative approach for calculating the electronic flexoelectric tensor consists in employing the time-dependent Schrödinger equation rewritten in curvilinear coordinates [13]. These coordinates are identified by the frame that is comoving with the atoms. In such a frame, the atoms do not move by construction, and all the information on the perturbation is encoded in the macroscopic displacement field and its gradients (e.g., the metric tensor). For this reason, we identify the aforementioned representation of the acoustic phonon as a "metric perturbation"; indeed, this constitutes a generalization of the metric tensor formulation of the uniform strain [8] to a spatially modulated perturbation. The curvilinear frame is particularly convenient because it naturally separates the static and the dynamic contributions to the electronic flexoelectric tensor. Thus, one can readily use the metric perturbation to calculate the static contribution directly, and thereby eliminate the need for any post-processing step connected with the diamagnetic correction. Moreover, the metric perturbation is a computationally much more efficient approach to the calculation of the clamped-ion flexoelectric tensor since it avoids the sum over individual sublattices which is implicit in the phonon approach of Ref. [12]. A practical calculation of the flexoelectric tensor that takes full advantage of the curvilinear coordinates, however, has not yet been attempted.

Part of the reason lies in some points of principle that were left unresolved in earlier works. First, the formalism of Ref. [13] was derived under the assumption of an all-electron description of the electronic structure, where the atoms are treated as point charges. This is clearly ill suited to a numerical implementation based on a plane-wave basis set. Prior to its practical use, the formalism needs to be generalized to the treatment of separable atomic pseudopotentials in the Kleinman-Bylander [18] form, at the very least. Second, the precise relationship between the first-order wave functions in the curvilinear and laboratory frames need to be established in order to firm up the conceptual foundations of the method. Based on earlier derivations [13], for example, we know the relationship between the relevant physical observables (current density, flexoelectric tensor, and so forth). However, this was obtained in an idealized context of a complete basis set and continuous Brillouin-zone integration. This is not enough to predict how results will converge as a function of the common computational parameters, nor whether such convergence will be at all different compared to the phonon approach [12].

Here, we address the above issues in full by deriving the missing pseudopotential terms in the metric wave perturbation. Remarkably, we establish a rigorous link between the response in the Cartesian and comoving frames, which shows that the respective first-order wave functions are related by a simple geometric contribution (i.e., one that can be expressed in terms of ground-state quantities). We analyze the implications of this result for the observables of interest in the present context (charge density and current), leading naturally to a stringent numerical validation strategy.

Based on the aforementioned results, we then proceed to the code implementation and testing of the monochromatic metric perturbation, identified by a (generally) incommensurate wave vector $\mathbf{q}$, in the context of DFPT. The wavefunction response to such a metric perturbation is then used as input for calculating the current-density response, as recently developed in Ref. [12]. The resulting methodology allows flexoelectric coefficients to be calculated with unprecedented accuracy and computational efficiency. In particular, our numerical tests clearly demonstrate that the present method yields faster convergence with respect to k-point mesh density and other computational parameters when compared with previous approaches. We rationalize this result in terms of the aforementioned relationship between the first-order wave functions in the curvilinear and laboratory frames.

From the formal point of view, this work also establishes a direct link between the perturbative treatment of phonon and uniform strain perturbations, which previously have been regarded as two conceptually distinct subareas of DFPT.

The paper is organized as follows. In Sec. II we start by briefly motivating the metric perturbation in the context of flexoelectricity. We then analyze in depth its connections with the established phonon and strain perturbations, while highlighting a number of important technical details related to the code implementation. In Sec. III we present the results of our numerical tests, which we perform in the context of DFPT by calculating, among other properties, the flexoelectric tensor of selected representative crystals. Finally, in Sec. IV, we present our summary and conclusions.

\section{THEORY}

\section{A. Flexoelectric tensor}

The main motivation for the development and implementation of the metric perturbation comes from the practical calculation of the clamped-ion (i.e., purely electronic) flexoelectric tensor. Thus, to frame our arguments, in this section we shall start by briefly reviewing the existing $[9,10,12]$ theory of bulk flexoelectricity and, in particular, the phonon-based currentdensity approach of Ref. [12]. Subsequently, we shall point out the advantages of the metric perturbation, which we shall describe in further detail in Sec. II B.

The bulk flexoelectric (FxE) coefficients are given by

$$
\mu_{\alpha \beta, \omega \nu}^{\mathrm{I}}=\frac{d P_{\alpha}}{d \eta_{\beta, \omega \nu}},
$$

where $P_{\alpha}$ is the polarization in direction $\alpha$, and

$$
\eta_{\beta, \omega v}=\frac{\partial^{2} u_{\beta}}{\partial r_{\omega} \partial r_{v}}
$$

(i.e., defined as the second gradient of the displacement field $\left.u_{\beta}\right)$. Alternatively, the FxE tensor can be written in type-II 
form as

$$
\mu_{\alpha \beta, \omega \nu}^{\mathrm{II}}=\frac{d P_{\alpha}}{d \varepsilon_{\omega \nu, \beta}}
$$

where $\varepsilon_{\omega \nu, \beta}=\partial \varepsilon_{\omega \nu} / \partial r_{\beta}$ is the first gradient of the symmetrized strain tensor,

$$
\varepsilon_{\omega v}=\frac{1}{2}\left(\frac{\partial u_{\omega}}{\partial r_{v}}+\frac{\partial u_{v}}{\partial r_{\omega}}\right)=\frac{h_{\omega \nu}+h_{v \omega}}{2}
$$

( $h_{\omega v}=\partial u_{\omega} / \partial r_{v}$ is the unsymmetrized strain, also known as deformation gradient). Choosing one or the other representation is a matter of convenience, as the independent entries of $\boldsymbol{\mu}^{\mathrm{I}}$ and $\boldsymbol{\mu}^{\mathrm{II}}$ are linearly related to one another. Neither of the two definitions [Eq. (1) or (3)], however, lends itself easily to a direct numerical implementation, as both involve an unbounded perturbation that breaks the translational symmetry of the lattice.

\section{Phonons}

To address this issue, Refs. $[9,10]$ based their formalism on the cell average of the microscopic polarization response to a monochromatic atomic distortion pattern

$$
\bar{P}_{\alpha, \kappa \beta}^{\mathbf{q}}=\frac{1}{\Omega} \int_{\text {cell }} d^{3} r e^{-i \mathbf{q} \cdot \mathbf{r}} \frac{\partial P_{\alpha}(\mathbf{r})}{\partial \lambda_{\kappa \beta}},
$$

where the perturbation consists in a modulated displacement of the sublattice $\kappa$ at the cell $l$ along the direction $\beta\left(\mathbf{R}_{l \kappa}\right.$ indicates the unperturbed lattice sites):

$$
u_{\kappa \beta}^{l}=\lambda_{\kappa \beta} e^{i \mathbf{q} \cdot \mathbf{R}_{l \kappa}} .
$$

The clamped-ion type-I flexoelectric coefficients can then be written as the second gradient with respect to the wave vector $\mathbf{q}$ of the aforementioned polarization response $[9,10]$

$$
\mu_{\alpha \beta, \omega \nu}^{\mathrm{I}}=-\frac{1}{2} \sum_{\kappa} \frac{\partial^{2} \bar{P}_{\alpha, \kappa \beta}^{\mathbf{q}}}{\partial q_{\omega} \partial q_{\nu}} .
$$

(Throughout this section we shall consistently use the type-I representation since it is the most convenient for performing the calculations, and we shall drop the superscript for conciseness. However, when presenting our results in Sec. IIIE, we shall switch to type-II form, following the conventions of earlier works.) Based on the current-density implementation of Ref. [12], one can write $\bar{P}_{\alpha, \kappa \beta}^{\mathbf{q}}$ as a second-order matrix element

$$
\bar{P}_{\alpha, \kappa \beta}^{\mathbf{q}}=\frac{4}{N_{k}} \sum_{n \mathbf{k}}\left\langle u_{n \mathbf{k}}\left|\hat{\mathcal{J}}_{\alpha}^{\mathbf{k}, \mathbf{q}}\right| \delta u_{n \mathbf{k}, \mathbf{q}}^{\tau_{\kappa \beta}}\right\rangle
$$

involving the ground-state Bloch orbitals $\left|u_{n \mathbf{k}}\right\rangle$, the currentdensity operator $\hat{\mathcal{J}}_{\alpha}^{\mathbf{k}, \mathbf{q}}$, and the adiabatic [12] wave-function response to the perturbation of Eq. (6), $\left|\delta u_{n \mathbf{k}, \mathbf{q}}^{\tau_{\kappa \beta}}\right\rangle$. (The latter is defined, in the context of adiabatic perturbation theory, as the change in the wave function to first order in the rate of change of the phonon mode amplitude.) In Ref. [12], some of us have implemented and tested Eq. (8), and used it to calculate the flexoelectric coefficients via Eq. (7); we refer the interested reader to that work for the technical details.

\section{Rotation gradients}

Many types of strain gradient involve a spatial variation in the local rotation as described by the antisymmetric part of the deformation gradient $R_{\omega v}=\left(\partial u_{\omega} / \partial r_{v}-\partial u_{v} / \partial r_{\omega}\right) / 2$. It has recently become clear $[12,13]$ that rotation gradients produce, in addition to other effects, a divergenceless currentdensity field, which contributes to the bulk flexoelectric tensor proportionally to the diamagnetic susceptibility of the material [13]. These contributions are implicitly present in Eq. (7); however, since they ultimately will cancel out with an equal and opposite surface term that originates from the same physical mechanism, it is best to subtract them once and for all. We can do this by defining the effective (type-I) flexoelectric tensor

$$
\mu_{\alpha \beta, \omega \nu}^{\prime}=\mu_{\alpha \beta, \omega \nu}-\frac{1}{2} \sum_{\gamma \lambda}\left(\epsilon^{\alpha \omega \gamma} \epsilon^{\beta \lambda \nu}+\epsilon^{\alpha \nu \gamma} \epsilon^{\beta \lambda \omega}\right) \chi_{\gamma \lambda}^{\operatorname{mag}},
$$

where $\epsilon$ is the Levi-Civita symbol and $\chi_{\gamma \lambda}^{\mathrm{mag}}=\partial M_{\gamma} / \partial H_{\lambda}$ is the magnetic susceptibility tensor ( $\mathbf{M}$ is the magnetization and $\mathbf{H}$ is the magnetic field). This was the approach taken in Ref. [12], where an independent calculation of $\chi_{\gamma \lambda}^{\mathrm{mag}}$ was performed in order to the obtain $\mu_{\alpha \beta, \omega \nu}^{\prime}$.

\section{Metric response}

The procedure described in Secs. II A 1 and II A 2 presents two drawbacks. First, an individual phonon response calculation needs to be performed for each sublattice $\kappa$. Second, an additional calculation of $\chi_{\gamma \lambda}^{\mathrm{mag}}$ needs to be performed. Both drawbacks were resolved in Ref. [13] by establishing an alternative formulation of the polarization response to an acoustic phonon

$$
\bar{P}_{\alpha, \beta}^{\prime \mathbf{q}}=\frac{4}{N_{k}} \sum_{n \mathbf{k}}\left\langle u_{n \mathbf{k}}\left|\hat{\mathcal{J}}_{\alpha}^{\mathbf{k}, \mathbf{q}}\right| \delta u_{n \mathbf{k}, \mathbf{q}}^{(\beta)}\right\rangle .
$$

Here, in contrast to Eq. (8), the ket is the adiabatic response to a metric wave, i.e., an acoustic phonon perturbation described in the comoving frame. Thus, the contributions from the individual atomic sublattices have implicitly been summed over. Furthermore, the resulting polarization is already free from the rotation-gradient contribution described in the previous paragraph, and therefore we can directly write

$$
\mu_{\alpha \beta, \omega \nu}^{\prime}=-\frac{1}{2} \frac{\partial^{2} \bar{P}_{\alpha, \beta}^{\prime \mathbf{q}}}{\partial q_{\omega} \partial q_{\nu}}
$$

eliminating the need for an explicit calculation of $\chi_{\gamma \lambda}^{\mathrm{mag}}$. Note that the metric formalism introduces another technical simplification at the level of the current-density operator. In the phonon case, an expression for $\hat{\mathcal{J}}_{\alpha}^{\mathbf{k}, \mathbf{q}}$ that is correct up to second order in $\mathbf{q}$ was needed [12]. Here, by contrast, since the wave-function response vanishes at $\mathbf{q}=0, \hat{\mathcal{J}}_{\alpha}^{\mathbf{k}, \mathbf{q}}$ is only needed up to first order,

$$
\hat{\mathcal{J}}_{\alpha}^{\mathbf{k}, \mathbf{q}}=-\left(\hat{p}_{\alpha}^{\mathbf{k}}+\frac{q_{\alpha}}{2}+\frac{\partial \hat{V}_{\mathrm{NL}}^{\mathbf{k}}}{\partial k_{\alpha}}+\frac{1}{2} \sum_{\gamma=1}^{3} q_{\gamma} \frac{\partial^{2} \hat{V}_{\mathrm{NL}}^{\mathbf{k}}}{\partial k_{\alpha} \partial k_{\gamma}}\right),
$$

where $\hat{p}_{\alpha}^{\mathbf{k}}=-i \hat{\nabla}_{\alpha}+k_{\alpha}$ is the cell-periodic momentum operator and $\hat{V}_{\mathrm{NL}}^{\mathbf{k}}=e^{i \mathbf{k} \cdot \hat{\mathbf{r}}} \hat{V}_{\mathrm{NL}} e^{-i \mathbf{k} \cdot \hat{\mathbf{r}}}$, where $\hat{V}_{\mathrm{NL}}$ nonlocal external 


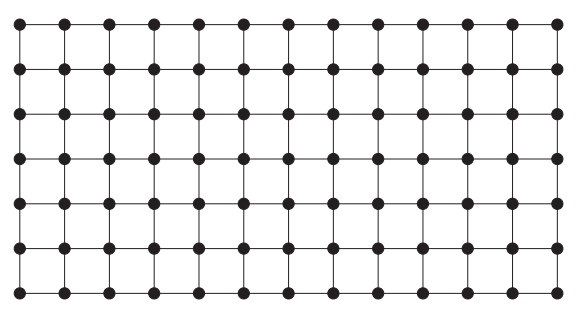

(a)

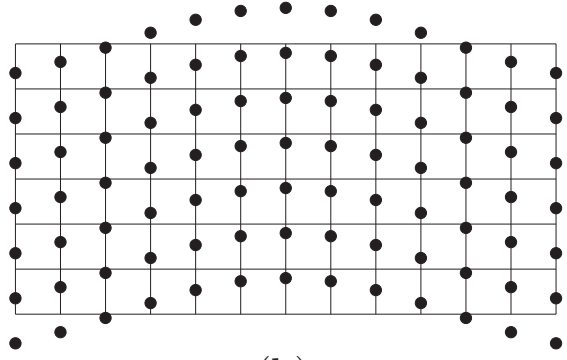

(b)

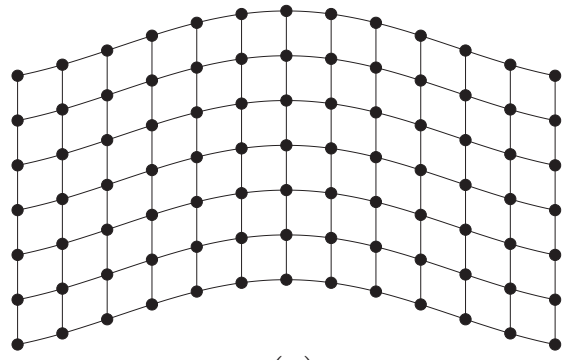

(c)

FIG. 1. Illustration of the coordinate transformation to the comoving frame. (a) Unperturbed crystal lattice; black circles represent the atomic sites, horizontal and vertical lines represent the coordinate system. (b) Transverse acoustic phonon in the laboratory frame. (c) The same phonon in the curvilinear frame; note that the atoms do not move in this coordinate system: the mechanical deformation is described via the metric.

potential operator. In this work, we will use the "straight-line path" form of the current operator $[19,20]$, although such a choice is irrelevant for materials with cubic symmetry [12].

In the following, we shall delve into the technical details of the metric perturbation within the general framework of DFPT.

\section{B. Metric perturbation}

The starting ingredient for the metric perturbation is a "clamped-ion" acoustic phonon. This is a collective lattice mode where all atoms are perturbed according to Eq. (6) by using the same displacement amplitude for all sublattices, $\lambda_{\kappa \beta}=\lambda_{\beta}$. Next, we describe such a monochromatic acoustic wave in the curvilinear frame that is comoving with the atoms (Fig. 1). This means that we combine the aforementioned displacement pattern with a simultaneous coordinate transformation that brings every atom back to its original position

$$
x_{\beta}(\xi)=\xi_{\beta}+\lambda_{\beta} e^{i \xi \cdot \mathbf{q}} .
$$

It is easy to verify that in the curvilinear reference, spanned by $\xi$, the atoms do not move; the perturbation now concerns the metric of the deformation.

The unperturbed Kohn-Sham Hamiltonian reads as

$$
\hat{H}^{(0)}=\hat{T}^{(0)}+\hat{V}^{\mathrm{Hxc},(0)}+\hat{V}^{\mathrm{psp},(0)},
$$

where $\hat{T}^{(0)}, \hat{V}^{\mathrm{Hxc},(0)}$, and $\hat{V}^{\mathrm{psp},(0)}$ are the kinetic, exchangecorrelation, and pseudopotential terms, respectively.

The latter consists in a local and a separable contribution

$$
V^{\mathrm{psp},(0)}\left(\mathbf{r}, \mathbf{r}^{\prime}\right)=V^{\mathrm{loc},(0)}(\mathbf{r}) \delta\left(\mathbf{r}-\mathbf{r}^{\prime}\right)+V^{\mathrm{sep},(0)}\left(\mathbf{r}, \mathbf{r}^{\prime}\right),
$$

both written as lattice sums of individual atomic contributions, e.g.,

$$
V^{\mathrm{loc},(0)}(\mathbf{r})=\sum_{l \kappa} v_{\kappa}^{\mathrm{loc}}\left(\mathbf{r}-\mathbf{R}_{l \kappa}\right),
$$

where $\mathbf{R}_{l \kappa}=\mathbf{R}_{l}+\boldsymbol{\tau}_{\kappa}$. The separable pseudopotential term is written in the Kleinman-Bylander (KB) form

$$
V^{\mathrm{sep},(0)}\left(\mathbf{r}, \mathbf{r}^{\prime}\right)=\sum_{l \kappa \mu} e_{\mu \kappa} \zeta_{\mu \kappa}\left(\mathbf{r}-\mathbf{R}_{l \kappa}\right) \zeta_{\mu \kappa}^{*}\left(\mathbf{r}^{\prime}-\mathbf{R}_{l \kappa}\right),
$$

where $\zeta_{\mu \kappa}(\mathbf{r})$ are the KB projectors, indexed by $\mu$, and $e_{\mu \kappa}$ are the corresponding coefficients.
Subsequently to the change of coordinates, we shall write the "static" first-order Hamiltonian in the curvilinear frame as

$$
\hat{\mathcal{H}}_{\mathbf{k}, \mathbf{q}}^{(\beta)}=\hat{H}_{\mathbf{k}, \mathbf{q}}^{(\beta)}+\hat{V}_{\mathbf{q}}^{(\beta)},
$$

i.e., as the sum of an "external potential" $\hat{H}_{\mathbf{k}, \mathbf{q}}^{(\beta)}$ plus a selfconsistent contribution

$$
V_{\mathbf{q}}^{(\beta)}(\mathbf{r})=\int d^{3} r^{\prime} K_{\mathrm{Hxc}}\left(\mathbf{r}, \mathbf{r}^{\prime}\right) e^{i \mathbf{q} \cdot\left(\mathbf{r}^{\prime}-\mathbf{r}\right)} n_{\mathbf{q}}^{(\beta)}\left(\mathbf{r}^{\prime}\right),
$$

that depends on the first-order charge density

$$
n_{\mathbf{q}}^{(\beta)}(\mathbf{r})=\frac{4}{N_{\mathbf{k}}} \sum_{m \mathbf{k}}\left\langle u_{m \mathbf{k}}^{(0)} \mid \mathbf{r}\right\rangle\left\langle\mathbf{r} \mid u_{m \mathbf{k}, \mathbf{q}}^{(\beta)}\right\rangle,
$$

via the Hartree and exchange-correlation kernel

$$
K_{\mathrm{Hxc}}\left(\mathbf{r}, \mathbf{r}^{\prime}\right)=\left.\frac{\delta V_{\mathrm{Hxc}}(\mathbf{r})}{\delta n\left(\mathbf{r}^{\prime}\right)}\right|_{n^{(0)}}=\left.\frac{\delta^{2} E_{\mathrm{Hxc}}}{\delta n(\mathbf{r}) \delta n\left(\mathbf{r}^{\prime}\right)}\right|_{n^{(0)}} .
$$

In contrast to most perturbations, however, the external potential here takes contributions from all individual pieces of the Hamiltonian, including the kinetic, pseudopotential, Hartree, and exchange-correlation terms. (The situation is analogous to the strain perturbation introduced by Hamann et al. [8], for which the same considerations hold.) In particular, loosely following Ref. [13], we shall write

$$
\hat{H}_{\mathbf{k}, \mathbf{q}}^{(\beta)}=\hat{T}_{\mathbf{k}, \mathbf{q}}^{(\beta)}+\hat{V}_{\mathbf{k}, \mathbf{q}}^{\mathrm{psp},(\beta)}+\hat{V}_{\mathbf{q}}^{\mathrm{H} 0,(\beta)}+\hat{V}_{\mathbf{q}}^{\mathrm{XC} 0,(\beta)}+\hat{V}_{\mathbf{q}}^{\mathrm{geom},(\beta)} .
$$

In Eq. (22),

$$
\hat{T}_{\mathbf{k}, \mathbf{q}}^{(\beta)}=-\frac{i}{2}\left[\left(\hat{p}_{\mathbf{k} \beta}+q_{\beta}\right) \mathbf{q} \cdot \hat{\mathbf{p}}_{\mathbf{k}}+\left(\hat{\mathbf{p}}_{\mathbf{k}}+\mathbf{q}\right) \cdot \mathbf{q} \hat{p}_{\mathbf{k} \beta}\right]
$$

is the kinetic term $\left(\hat{p}_{\mathbf{k} \beta}=-i \partial / \partial \xi_{\beta}+k_{\beta}\right.$ is the canonical momentum operator in curvilinear space). For notational purposes, we shall write the remainder of the contributions as matrix elements on two plane waves, e.g.,

$$
W_{\mathbf{k}, \mathbf{q}}^{(\beta)}\left(\mathbf{G}, \mathbf{G}^{\prime}\right)=\left\langle\mathbf{G}+\mathbf{k}+\mathbf{q}\left|\hat{W}_{\mathbf{k}, \mathbf{q}}^{(\beta)}\right| \mathbf{G}^{\prime}+\mathbf{k}\right\rangle
$$

for an arbitrary operator $\hat{W}$.

Regarding the pseudopotential term, we operate the same decomposition as in Eq. (15),

$$
V_{\mathbf{k}, \mathbf{q}}^{\mathrm{psp},(\beta)}\left(\mathbf{G}, \mathbf{G}^{\prime}\right)=V_{\mathbf{q}}^{\mathrm{loc},(\beta)}\left(\mathbf{G}-\mathbf{G}^{\prime}\right)+V_{\mathbf{k}, \mathbf{q}}^{\mathrm{sep},(\beta)}\left(\mathbf{G}, \mathbf{G}^{\prime}\right),
$$


i.e., we write it as the sum of a local

$$
V_{\mathbf{q}}^{\mathrm{loc},(\beta)}(\mathbf{G})=i G_{\beta} \frac{1}{\Omega} \sum_{\kappa} e^{-i \mathbf{G} \cdot \boldsymbol{\tau}_{\kappa}} v_{\kappa}^{\mathrm{loc}}(\mathbf{G})-i\left(G_{\beta}+q_{\beta}\right) \frac{1}{\Omega} \sum_{\kappa} e^{-i \mathbf{G} \cdot \boldsymbol{\tau}_{\kappa}} v_{\kappa}^{\text {loc }}(\mathbf{G}+\mathbf{q}),
$$

and a nonlocal contribution

$$
\begin{aligned}
V_{\mathbf{k}, \mathbf{q}}^{\mathrm{sep},(\beta)}\left(\mathbf{G}, \mathbf{G}^{\prime}\right)= & \frac{1}{\Omega} \sum_{\mu \kappa} e_{\mu \kappa} e^{-i\left(\mathbf{G}-\mathbf{G}^{\prime}\right) \cdot \tau_{\kappa}}\left\{i\left(G_{\beta}+k_{\beta}+\frac{q_{\beta}}{2}\right) \zeta_{\mu \kappa}(\mathbf{G}+\mathbf{k}) \zeta_{\mu \kappa}^{*}\left(\mathbf{G}^{\prime}+\mathbf{k}\right)-i\left(G_{\beta}-G_{\beta}^{\prime}+q_{\beta}\right)\right. \\
& \left.\times \zeta_{\mu \kappa}(\mathbf{G}+\mathbf{k}+\mathbf{q}) \zeta_{\mu \kappa}^{*}\left(\mathbf{G}^{\prime}+\mathbf{k}\right)-i\left(G_{\beta}^{\prime}+k_{\beta}+\frac{q_{\beta}}{2}\right) \zeta_{\mu \kappa}(\mathbf{G}+\mathbf{k}+\mathbf{q}) \zeta_{\mu \kappa}^{*}\left(\mathbf{G}^{\prime}+\mathbf{k}+\mathbf{q}\right)\right\},
\end{aligned}
$$

where $\zeta_{\mu \kappa}(\mathbf{G}+\mathbf{k})$ indicates the Fourier components of the $\mathrm{KB}$ projectors.

The two terms

$$
\begin{gathered}
V_{\mathbf{q}}^{\mathrm{H} 0,(\beta)}(\mathbf{G})=4 \pi i\left(-\frac{G_{\beta}+q_{\beta}}{|\mathbf{G}+\mathbf{q}|^{2}}+\frac{G_{\beta}}{G^{2}}\right) n^{(0)}(\mathbf{G}), \\
V_{\mathbf{q}}^{\mathrm{XC} 0,(\beta)}(\mathbf{G})=-i q_{\beta} V^{\mathrm{xc},(0)}(\mathbf{G})
\end{gathered}
$$

are the "geometric" (i.e., only depending on the unperturbed quantity $n^{(0)}$ ) contributions to the Hartree $(\mathrm{H})$ and exchangecorrelation (XC) potentials, respectively. Finally,

$$
V_{\mathbf{q}}^{\text {geom, }(\beta)}=-\frac{i}{4} q_{\beta} q^{2}
$$

is an additional geometric potential originating from the change of coordinates, which we introduce here for completeness (this structureless potential is irrelevant for either the uniform strain or the strain-gradient response, as it is of third order in $\mathbf{q}$ ).

Explicit derivations of most of the above expressions can be found in Ref. [13]. Regarding the pseudopotential pieces, which have been derived here, some additional details can be found in the Appendix.

Based on the above, it is now easy to demonstrate the following points:

(i) For an arbitrary $\mathbf{q}, V_{\mathbf{q}}^{\mathrm{H} 0,(\beta)}(\mathbf{G})$ exactly matches the metric contribution to the electrostatic potential as derived in Ref. [13].

(ii) The external perturbation $\hat{H}_{\mathbf{k}, \mathbf{q}}^{(\beta)}$ identically vanishes in the limit $\mathbf{q}=0$.

(iii) The first $\mathbf{q}$ gradient of the above expressions recovers the Hamann-Wu-Rabe-Vanderbilt (HWRV) [8] treatment of the uniform strain

$$
\hat{\mathcal{H}}_{\mathbf{k}, \gamma}^{(\beta)}=i \hat{\mathcal{H}}_{\mathbf{k}}^{(\beta \gamma)}
$$

and is symmetric under $\beta \gamma$ exchange.

We have, therefore, achieved the desired generalization of the HWRV metric tensor formalism to a monochromatic displacement wave of arbitrary $\mathbf{q}$.

\section{Relationship to the response in the laboratory frame}

In this section, we shall establish the explicit link between the metric perturbation described in the previous subsection (which, as we said, is defined in the comoving frame and reduces to a uniform strain perturbation in the long-wave limit) and the familiar phonon perturbation, which is defined in the laboratory frame. In particular, we shall show that the corresponding response functions ("metric" versus "phonon") differ by a geometric piece that depends on the groundstate orbitals only. These analytical results will prove to be important for testing our numerical implementation, as we shall see shortly. They also provide an interesting formal unification of two areas of DFPT (related to the response to phonons and strains, respectively) that were formerly regarded as conceptually distinct.

The first-order external potential for a phonon perturbation consists of a local potential plus a separable contribution

$$
H_{\mathbf{k}, \mathbf{q}}^{\tau_{\kappa \beta}}\left(\mathbf{G}, \mathbf{G}^{\prime}\right)=V_{\mathbf{q}}^{\text {loc, } \tau_{\kappa \beta}}\left(\mathbf{G}-\mathbf{G}^{\prime}\right)+V_{\mathbf{k}, \mathbf{q}}^{\mathrm{sep}, \tau_{\kappa \beta}}\left(\mathbf{G}, \mathbf{G}^{\prime}\right),
$$

where

$$
\begin{aligned}
V_{\mathbf{q}}^{\text {loc, } \tau_{\kappa \beta}}(\mathbf{G})= & -i\left(G_{\beta}+q_{\beta}\right) \frac{1}{\Omega} e^{-i \mathbf{G} \cdot \tau_{\kappa}} v_{\kappa}^{\text {loc }}(\mathbf{G}+\mathbf{q}), \\
V_{\mathbf{k}, \mathbf{q}}^{\text {sep }, \tau_{\kappa \beta}}\left(\mathbf{G}, \mathbf{G}^{\prime}\right)= & -i\left(G_{\beta}+q_{\beta}-G_{\beta}^{\prime}\right) \frac{1}{\Omega} \sum_{\mu} e^{-i\left(\mathbf{G}-\mathbf{G}^{\prime}\right) \cdot \tau_{\kappa}} \\
& \times e_{\mu \kappa} \zeta_{\mu \kappa}(\mathbf{k}+\mathbf{q}+\mathbf{G}) \zeta_{\mu \kappa}^{*}\left(\mathbf{k}+\mathbf{G}^{\prime}\right) .
\end{aligned}
$$

Note that in Eqs. (33) and (34), the structure factors differ slightly from those that are commonly implemented in DFPT (e.g., Ref. [21]), which read as $e^{-i(\mathbf{G}+\mathbf{q}) \cdot \tau_{\kappa}}$ and $e^{-i\left(\mathbf{G}-\mathbf{G}^{\prime}+\mathbf{q}\right) \cdot \boldsymbol{\tau}_{\kappa}}$, respectively. This difference is a consequence of the fact that here we have introduced an extra phase $e^{i \boldsymbol{q} \cdot \tau_{\kappa}}$ into the monochromatic phonon perturbation [10].

In the laboratory frame, an acoustic phonon perturbation can be readily constructed as a sublattice sum of the above:

$$
H_{\mathbf{k}, \mathbf{q}}^{u_{\beta}}\left(\mathbf{G}, \mathbf{G}^{\prime}\right)=\sum_{\kappa} H_{\mathbf{k}, \mathbf{q}}^{\tau_{\kappa \beta}}\left(\mathbf{G}, \mathbf{G}^{\prime}\right)
$$

Here and in the following, we use the symbol $u_{\beta}$ to indicate a laboratory-frame acoustic phonon perturbation, not to be confused with the corresponding metric perturbation labeled by $(\beta)$. The corresponding first-order wave functions satisfy the following Sternheimer equation [21]:

$$
\left(\hat{H}_{\mathbf{k}+\mathbf{q}}^{(0)}+a \hat{P}_{\mathbf{k}+\mathbf{q}}-\epsilon_{m \mathbf{k}}^{(0)}\right)\left|u_{m \mathbf{k}, \mathbf{q}}^{u_{\beta}}\right\rangle=-\hat{Q}_{\mathbf{k}+\mathbf{q}} \hat{\mathcal{H}}_{\mathbf{k}, \mathbf{q}}^{u_{\beta}}\left|u_{m \mathbf{k}}^{(0)}\right\rangle,
$$

where $\hat{\mathcal{H}}_{\mathbf{k}, \mathbf{q}}^{u_{\beta}}$ is, as usual, the self-consistent counterpart of $\hat{H}_{\mathbf{k}, \mathbf{q}}^{u_{\beta}}, \hat{P}_{\mathbf{k}+\mathbf{q}}$, and $\hat{Q}_{\mathbf{k}+\mathbf{q}}=1-\hat{P}_{\mathbf{k}+\mathbf{q}}$ are the projectors on the valence and conduction band manifolds, respectively, and $a$ is a positive constant that makes the operator in the round brackets nonsingular [22]. Note that an acoustic phonon physically reduces to a rigid translation of the whole crystal lattice at 
$\mathcal{O}\left(q^{0}\right)$. At $\mathcal{O}\left(q^{1}\right)$ and $\mathcal{O}\left(q^{2}\right)$, respectively, it should provide complete information about the response to a uniform strain and strain-gradient deformation.

To see the relationship between the laboratory and curvilinear frame pictures, it is convenient to take one step back, and consider the first-order Hamiltonians in the original Hilbert space, i.e., without factoring out the incommensurate phases that belong either to the Bloch orbitals or to the first-order Hamiltonian. (Recall that the first-order Hamiltonian $\hat{\mathcal{H}}^{\lambda}$ is related to its periodic part in momentum space as $\hat{\mathcal{H}}_{\mathbf{k}, \mathbf{q}}^{\lambda}=$ $\left.e^{-i(\mathbf{k}+\mathbf{q}) \cdot \mathbf{r}} \hat{\mathcal{H}}^{\lambda} e^{i \mathbf{k} \cdot \mathbf{r}}\right)$. We shall postulate (and later prove that it is consistent with the results derived so far) the relationship

$$
\hat{\mathcal{H}}^{u_{\beta}}=\hat{\mathcal{H}}^{(\beta)}+i\left[\hat{H}^{(0)}, \frac{1}{2}\left(e^{i \mathbf{q} \cdot \mathbf{r}} \hat{p}_{\beta}+\hat{p}_{\beta} e^{i \mathbf{q} \cdot \mathbf{r}}\right)\right] .
$$

One can recognize in the commutator the gauge-field contribution to the perturbation in curvilinear coordinates discussed in Ref. [13]:

$$
\hat{\mathcal{H}}^{\dot{\lambda}_{\beta}}(\mathbf{q})=-\frac{1}{2}\left(e^{i \mathbf{q} \cdot \mathbf{r}} \hat{p}_{\beta}+\hat{p}_{\beta} e^{i \mathbf{q} \cdot \mathbf{r}}\right) .
$$

[By taking the momentum-space representation of the above operator $\hat{\mathcal{H}}_{\mathbf{k}, \mathbf{q}}^{\dot{\lambda}_{\beta}}=e^{-i(\mathbf{k}+\mathbf{q}) \cdot \mathbf{r}} \hat{\mathcal{H}}^{\dot{\lambda}_{\beta}}(\mathbf{q}) e^{i \mathbf{k} \cdot \mathbf{r}}$, one readily recovers Eq. (90) of Ref. [13]]. This clarifies the physical interpretation of Eq. (37) as being closely linked to the coordinate change discussed in Ref. [13]. Then, after reverting to our previous notation, the relation between laboratory-frame and metric responses in Eq. (37) becomes

$$
\hat{\mathcal{H}}_{\mathbf{k}, \mathbf{q}}^{u_{\beta}}=\hat{\mathcal{H}}_{\mathbf{k}, \mathbf{q}}^{(\beta)}+i \hat{H}_{\mathbf{k}+\mathbf{q}}^{(0)}\left(\hat{p}_{\mathbf{k} \beta}+\frac{q_{\beta}}{2}\right)-i\left(\hat{p}_{\mathbf{k} \beta}+\frac{q_{\beta}}{2}\right) \hat{H}_{\mathbf{k}}^{(0)} .
$$

The correctness of this result can be verified by comparing the explicit formulas for the perturbed Hamiltonians piece by piece. In particular, the second and the third terms on the right-hand side of Eq. (39) precisely cancel the kinetic and geometric contributions in $\hat{\mathcal{H}}_{\mathbf{k}, \mathbf{q}}^{(\beta)}$, and they also account for the difference between the pseudopotential, Hartree, and XC terms in $\hat{\mathcal{H}}_{\mathbf{k}, \mathbf{q}}^{(\beta)}$ and $\hat{\mathcal{H}}_{\mathbf{k}, \mathbf{q}}^{u_{\beta}}$.

If we now plug Eq. (39) into (36), we obtain an analogous Sternheimer equation with $\hat{\mathcal{H}}_{\mathbf{k}, \mathbf{q}}^{(\beta)}$ replacing $\hat{\mathcal{H}}_{\mathbf{k}, \mathbf{q}}^{u_{\beta}}$, and with the laboratory-frame first-order wave functions related to the metric ones by

$$
\left|u_{m \mathbf{k}, \mathbf{q}}^{u_{\beta}}\right\rangle=\left|u_{m \mathbf{k}, \mathbf{q}}^{(\beta)}\right\rangle+\left|\Delta u_{m \mathbf{k}}^{\beta}\right\rangle,
$$

where $\left|\Delta u_{m \mathbf{k}}^{\beta}\right\rangle$ is a purely geometric (i.e., defined in terms of the ground-state orbitals only) contribution

$$
\left|\Delta u_{m \mathbf{k}}^{\beta}\right\rangle=-i \hat{Q}_{\mathbf{k}+\mathbf{q}}\left(\hat{p}_{\mathbf{k} \beta}+\frac{q_{\beta}}{2}\right)\left|u_{m \mathbf{k}}^{(0)}\right\rangle .
$$

This constitutes the main result of this section.

To illustrate its physical meaning it is useful, first of all, to calculate the contribution of $\left|\Delta u_{m \mathbf{k}, \mathbf{q}}^{\beta}\right\rangle$ to the firstorder electron density, and check whether it matches our expectations for the relationship between its laboratory-frame and curvilinear-frame representations. To this end, recall the definition of the density response to a generic perturbation $\lambda$ :

$$
n_{\mathbf{q}}^{\lambda}(\mathbf{r})=\frac{4}{N_{\mathbf{k}}} \sum_{n \mathbf{k}}\left\langle u_{n \mathbf{k}}^{(0)} \mid \mathbf{r}\right\rangle\left\langle\mathbf{r} \mid u_{n \mathbf{k}, \mathbf{q}}^{\lambda}\right\rangle .
$$

By combining Eq. (42) with (40), we find

$$
n_{\mathbf{q}}^{u^{\beta}}(\mathbf{r})=n_{\mathbf{q}}^{(\beta)}(\mathbf{r})+\Delta n_{\mathbf{q}}^{\beta}(\mathbf{r}),
$$

where $\Delta n_{\mathbf{q}}^{\beta}(\mathbf{r})$ is, again, a purely geometric object. One can arrive at an explicit formula after observing that $\hat{Q}_{\mathbf{k}+\mathbf{q}}=1-$ $\hat{P}_{\mathbf{k}+\mathbf{q}}$; this leads to two separate contributions to $\Delta n_{\mathbf{q}}^{\beta}(\mathbf{r})$. The part that contains the band projector $\hat{P}_{\mathbf{k}+\mathbf{q}}$ vanishes identically, which can be seen in the following way. Any physical scalar field must be real, which implies

$$
n_{-\mathbf{q}}^{(1)}(\mathbf{r})=n_{\mathbf{q}}^{(1) *}(\mathbf{r}) .
$$

Thus, we can write the contribution of $\hat{P}_{\mathbf{k}+\mathbf{q}}$ to $\Delta n_{\mathbf{q}}^{\beta}(\mathbf{r})$ as

$$
\begin{aligned}
& \sum_{m j} i\left\langle u_{m \mathbf{k}}^{(0)} \mid \mathbf{r}\right\rangle\left\langle\mathbf{r} \mid u_{j \mathbf{k}+\mathbf{q}}^{(0)}\right\rangle\left\langle u_{j \mathbf{k}+\mathbf{q}}^{(0)}\left|\left(\hat{p}_{\mathbf{k} \beta}+\frac{q_{\beta}}{2}\right)\right| u_{m \mathbf{k}}^{(0)}\right\rangle \\
& -\sum_{m j} i\left\langle u_{j \mathbf{k}-\mathbf{q}}^{(0)} \mid \mathbf{r}\right\rangle\left\langle\mathbf{r} \mid u_{m \mathbf{k}}^{(0)}\right\rangle\left\langle u_{m \mathbf{k}}^{(0)}\left|\left(\hat{p}_{\mathbf{k} \beta}-\frac{q_{\beta}}{2}\right)\right| u_{j \mathbf{k}-\mathbf{q}}^{(0)}\right\rangle .
\end{aligned}
$$

After operating a translation in $\mathbf{k}$ space on the second line (this is irrelevant, as the expression needs to be integrated over the whole Brillouin zone), the result manifestly vanishes.

We are left in Eq. (41) with just the contribution of the identity operator, which can be written as

$$
\Delta n_{\mathbf{q}}^{\beta}(\mathbf{r})=-\frac{\partial n^{(0)}(\mathbf{r})}{\partial r_{\beta}}-i q_{\beta} n^{(0)}(\mathbf{r}) .
$$

The form of Eq. (46) might appear puzzling at first sight, but in fact it accurately matches the known relationship between the charge-density responses in the curvilinear and Cartesian reference frames $[14,23]$. For example, at $\mathbf{q}=0$, we already know that the metric perturbation (and, as a consequence, the corresponding density response) must vanish,

$$
\hat{\mathcal{H}}_{\mathbf{k}, \mathbf{q}=0}^{(\beta)}=0, \quad n_{\mathbf{q}=0}^{(\beta)}(\mathbf{r})=0,
$$

since a uniform translation of the crystal has no effect in its own comoving reference frame. Also, by translational symmetry one must have, for the laboratory-frame perturbation

$$
\hat{\mathcal{H}}_{\mathbf{k}, \mathbf{q}=0}^{u_{\beta}}=i\left[\hat{H}_{\mathbf{k}}^{(0)}, \hat{p}_{\mathbf{k} \beta}\right],
$$

which implies that

$$
n_{\mathbf{q}=0}^{u^{\beta}}(\mathbf{r})=-\frac{\partial n^{(0)}(\mathbf{r})}{\partial r_{\beta}} .
$$

One can easily check that our formulas for $\hat{\mathcal{H}}_{\mathbf{k}, \mathbf{q}}^{(\beta)}$, together with the results (39), (43), and (46), are fully consistent with these requirements.

In other words, Eq. (46) corroborates our interpretation of the modified perturbation as a metric wave, where the atomic displacements are expressed as a local modification of the metric of space. From this perspective, $\left|\Delta u_{m \mathbf{k}, \mathbf{q}}^{\beta}\right\rangle$ is essential for ensuring that the first-order density response complies with the established transformation laws.

\section{Implementation considerations}

The formulas derived in the previous section are formal, but when implementing them we need to introduce 
approximations in order to make the calculations tractable. In particular, we make a set of choices concerning the discrete sampling of the Brillouin zone (BZ) with a finite mesh, and the plane-wave energy cutoff used in the wave-function expansion. It is therefore important to clarify which of the above relations remain exact in principle, once such a set of choices has been made, and which should be expected to show discrepancies (of course, these will diminish as more highly converged choices are made).

Our main focus will be on Eq. (46), describing the difference between the electron-density response to a phonon perturbation in the laboratory frame, already available within the existing DFPT implementations, and the metric response introduced in this work.

First of all, note that, in order to obtain Eq. (46), we have used the fact that the expression in Eq. (45) vanishes; this, in turn, relies on the fact that it must be integrated over the whole Brillouin zone. If the BZ is sampled by a discrete number of $\mathbf{k}$ points, then Eq. (45) is only approximately satisfied; in fact, one can see that it holds exactly only if the set of $\mathbf{k}$ points is invariant under a translation by $\mathbf{q}$, i.e., $\mathbf{q}$ is commensurate with the $\mathbf{k}$ points.

Next, as we shall see in the following, commensuration between $\mathbf{q}$ and the $\mathbf{k}$ mesh does not automatically guarantee that Eq. (46) is exact. To see why, it is useful to write the explicit expression for the charge-density difference as

$$
\begin{aligned}
\Delta n_{\mathbf{q}}^{\beta}(\mathbf{r}) & =-\frac{4 i}{N_{\mathbf{k}}} \sum_{m \mathbf{k}}\left\langle u_{m \mathbf{k}}^{(0)} \mid \mathbf{r}\right\rangle\left\langle\mathbf{r}\left|\hat{Q}_{\mathbf{k}+\mathbf{q}}\left(\hat{p}_{\mathbf{k} \beta}+\frac{q_{\beta}}{2}\right)\right| u_{m \mathbf{k}}^{(0)}\right\rangle \\
& =-\frac{4 i}{N_{\mathbf{k}}} \sum_{m \mathbf{k}}\left\langle u_{m \mathbf{k}}^{(0)} \mid \mathbf{r}\right\rangle\left\langle\mathbf{r}\left|\left(\hat{p}_{\mathbf{k} \beta}+\frac{q_{\beta}}{2}\right)\right| u_{m \mathbf{k}}^{(0)}\right\rangle,
\end{aligned}
$$

where $N_{\mathbf{k}}$ is the number of $\mathbf{k}$ points (a uniform mesh is assumed), and the second equality relies on the assumed commensuration between $\mathbf{q}$ and the mesh (see above discussion). Equation (50), however, only satisfies Eq. (46) in the limit of a complete plane-wave basis set, i.e., for an infinitely large plane-wave cutoff $E_{\text {cut }}$. In practice, a finite basis set is always used, which means that plane waves with a kinetic energy that is larger than $E_{\text {cut }}$ are discarded from the calculation. Crucially, the kinetic energy of a plane wave is calculated as $|\mathbf{G}+\mathbf{k}|^{2} / 2$, which implies that different $\mathbf{k}$ points are characterized by different cutoff spheres in reciprocal space, and hence by different basis sets. For example, the wave function $u_{\mathbf{k}}^{(0)}(\mathbf{r})$ has nonzero coefficients only inside a cutoff sphere centered in $-\mathbf{k}$, while the sphere of both the phonon and metric response functions $u_{\mathbf{k}, \mathbf{q}}^{(1)}(\mathbf{r})$ is centered in $-(\mathbf{k}+\mathbf{q})$ (see Fig. 2). Now, note that the function $\left|\Delta u_{m \mathbf{k}, \mathbf{q}}^{\beta}\right\rangle$ that we have used to define $\Delta n_{\mathbf{q}}^{\beta}(\mathbf{r})$ "belongs" to the point $\mathbf{k}+\mathbf{q}$, and hence it will not, in general, be represented on the same basis set as $\left|u_{m \mathbf{k}}^{(0)}\right\rangle$; this is the reason why Eq. (46) is generally violated when a finite $E_{\text {cut }}$ is used.

To illustrate this point more clearly, we can write the charge-density difference, as it is computed in practice starting from $n^{\beta}$ and $n^{u_{\beta}}$, as

$$
\Delta n_{\mathbf{q}}^{\beta}(\mathbf{r})=-\frac{4 i}{N_{\mathbf{k}}} \sum_{m \mathbf{k}}\left\langle u_{m \mathbf{k}}^{(0)} \mid \mathbf{r}\right\rangle\left\langle\mathbf{r}\left|\left(\hat{p}_{\mathbf{k} \beta}+\frac{q_{\beta}}{2}\right)\right| \tilde{u}_{m \mathbf{k}}^{(0)}\right\rangle .
$$

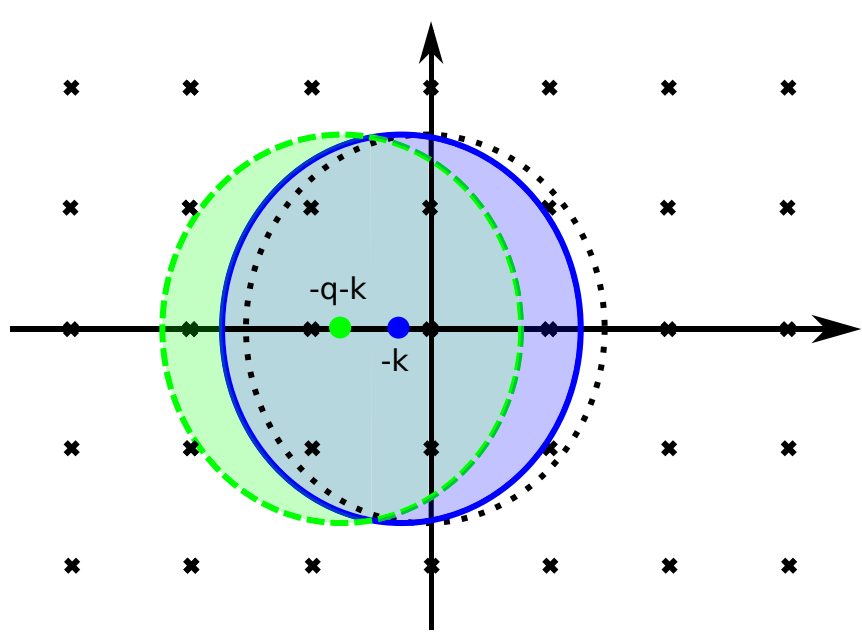

FIG. 2. Representation of the Fourier space in 2D. The small black crosses are the $\mathbf{G}$ vectors; the black dotted circle identifies the cutoff sphere centered on $\Gamma$; the blue continuous circle identifies nonzero Fourier coefficients of $u_{\mathbf{k}}^{(0)}$; and the dashed green circle identifies the Fourier coefficients of $\tilde{u}_{\mathbf{k}}^{(0)}$.

Here, $\tilde{u}_{m \mathbf{k}}^{(0)}(\mathbf{r})$ is the same as $u_{m \mathbf{k}}^{(0)}(\mathbf{r})$ in Eq. (50) except that it has nonzero Fourier components only in the intersection between the green and blue circles of Fig. 2, while $u_{m \mathbf{k}}^{(0)}(\mathbf{r})$ is defined inside the whole blue solid circle. Since the first-order wave functions are obtained through a self-consistent process, this error will propagate to the potentials and back to the density; thus, at the end of the calculation even the "revised" relationship (51) will not be exactly fulfilled. In any case, we can expect that the error will be roughly linear in $|\mathbf{q}|$, and should rapidly vanish upon increasing the plane-wave cutoff; we shall see that both expectations are nicely fulfilled in our tests. As we shall show shortly, this discrepancy between the phonon and metric approach results in a faster numerical convergence of the latter with respect to plane-wave cutoff and $k$-point sampling.

\section{RESULTS}

To test our implementation, we have compared the results of the metric perturbation against response functions that are already present in publicly available DFPT codes: the phonon perturbation [24] and the uniform strain perturbation [8]. The quantities that we used to gauge the accuracy of the implementation are either based on the first-order charge density (a fundamental linear-response quantity), or on the cell-averaged polarization. In particular, we have performed four independent tests:

(i) In Sec. III B, we compare the electron density response of a "clamped-ion acoustic phonon" to the electron density response to a metric perturbation, following the guidelines of Sec. II C.

(ii) In Sec. IIIC, we compare the electron density response of a uniform strain perturbation to the electron density response of a metric perturbation at first order in q. We have already demonstrated in Eq. (31) the relation that must hold between the uniform strain Hamiltonian and the metric perturbation. In the same way, the response density to a metric 
and the associated uniform strain perturbation are related by

$$
-i n_{\alpha}^{1, \beta}=n_{\alpha \beta}^{\text {strain }},
$$

where $n_{\alpha}^{1, \beta}$ is the first derivative of the microscopic metric perturbation response, $n^{1, \beta}$, respect to the $q_{\alpha}$. Note that, by time-reversal symmetry, $n_{\alpha}^{1, \beta}$ is a pure imaginary function.

(iii) In Sec. IIID, we compare the octupolar response calculated via the phonon to that from metric perturbation. The octupolar tensor components can be extracted via the long-wave expansion of the macroscopic (i.e., cell-integrated) charge-density response

$$
Q_{\delta}^{(3, \alpha \beta \gamma)}=\left.\int_{\text {cell }} d^{3} r \frac{\partial^{3} n_{\mathbf{q}}^{(\delta)}(\mathbf{r})}{\partial q_{\alpha} \partial q_{\beta} \partial q_{\gamma}}\right|_{\mathbf{q}=0},
$$

where $\delta$ indicates the atomic displacement direction. Clearly, since the geometrical term $\Delta n^{\beta}$ averages to zero, both the phonon and metric calculations must yield the same values of $Q_{\delta}^{(3, \alpha \beta \gamma)}$. The $q$ derivative can be performed by fitting the cell-integrated density as a function of $\mathbf{q}$ in a vicinity of $\mathbf{q}=0$, as described in Ref. [14]. Testing this quantity is particularly interesting in the context of this work because the longitudinal octupole $Q_{\mathrm{L}}=Q_{\alpha}^{(3, \alpha \alpha \alpha)}$ is directly related to the longitudinal FxE coefficient by $\mu_{\mathrm{L}}=Q_{\mathrm{L}} /(6 \Omega)$.

(iv) In Sec. III E, we compare FxE coefficients calculated via the phonon method [Eqs. (7)-(9)] and the metric wave method [Eqs. (10) and (11)].

Note that, whenever a 3D scalar field is involved (first and second tests), we shall use the "distance"

$$
d(f, g)=\frac{1}{\Omega} \int_{\text {cell }} d^{3} r|f(\mathbf{r})-g(\mathbf{r})|
$$

to gauge their overall difference, where functions $f$ and $g$ identify the left- and right-hand sides of the given relation that is to be verified.

\section{A. Computational setup}

We have used two types of systems for our benchmark tests in the following sections: isolated noble-gas atoms in large boxes and cubic bulk solids. Regarding the isolated atoms, we have tested three different noble gases: $\mathrm{He}, \mathrm{Ne}$, and $\mathrm{Kr}$. As for the cubic solids, we have used crystalline $\mathrm{Si}$ in the diamond structure, and the cubic perovskite phase of $\mathrm{SrTiO}_{3}$.

Our calculations are performed in the framework of density functional theory, using the local-density approximation (we have employed the Perdew-Wang 92 parametrization [25] of the exchange and correlation). The core-valence interactions are described by Troullier-Martins [26] normconserving pseudopotentials, which we have generated via the FHI98PP [27] code with the following electronic configurations: $\mathrm{He}=1 s^{2} ; \mathrm{Ne}=2 s^{2} 2 p^{6} ; \mathrm{Kr}=4 s^{2} 4 p^{6} ; \mathrm{Si}=3 s^{2} 3 p^{2}$; $\mathrm{Sr}=4 s^{2} 4 p^{5} s^{2} ; \mathrm{Ti}=3 s^{2} 3 p^{6} 3 d^{2} 4 s^{2} ; \mathrm{O}=2 s^{2} 2 p^{4}$. Note that the He pseudopotential only contains a local part.

The noble-gas atoms have been simulated in cubic boxes large enough to avoid interaction between the replicas. For the tests in Secs. III B, III C, and IIID, the size of such box are 5 bohrs for $\mathrm{He}, 7$ bohrs for $\mathrm{Ne}$, and 14 bohrs for $\mathrm{Kr}$, with (unless specified) Monkhorst-Pack (MP) $k$ meshes [28] of $8 \times$ $8 \times 8$ for $\mathrm{He}$ and $\mathrm{Ne}$, and $4 \times 4 \times 4$ for $\mathrm{Kr}$. For the calculation

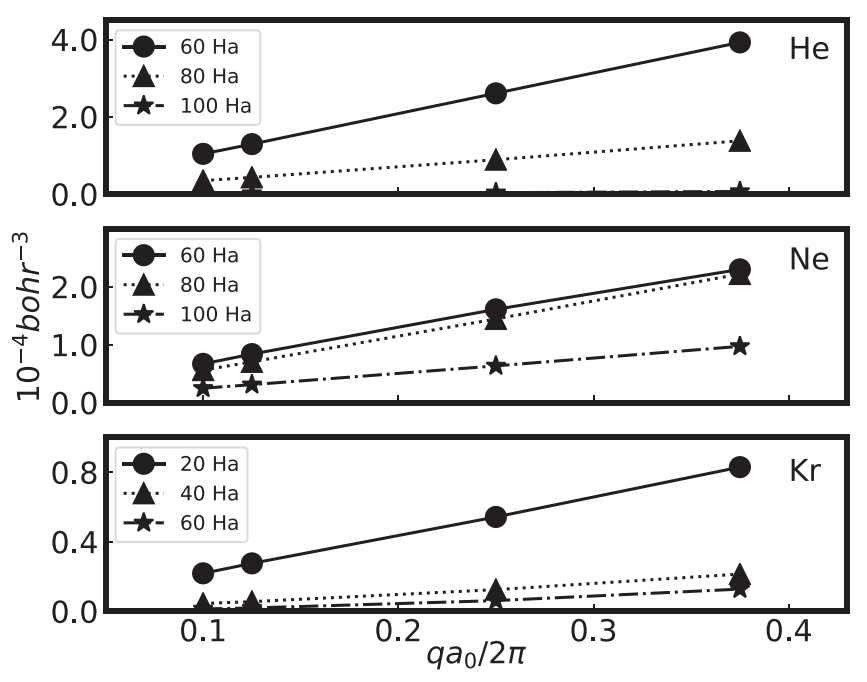

FIG. 3. Plot of $d\left(n_{\mathbf{q}}^{u^{\beta}}(\mathbf{r}), n_{\mathbf{q}}^{(\beta)}(\mathbf{r})+\Delta n_{\mathbf{q}}^{\beta}(\mathbf{r})\right)$ [cf. Eq. (54)] as a function of wave vector $\mathbf{q}$ (reduced coordinates), for different cutoffs. All the results refer to longitudinal perturbations. From top to bottom: $\mathrm{He}, \mathrm{Ne}$, and $\mathrm{Kr}$.

of flexoelectric constants in Sec. III E, 14-bohr boxes were used for all atoms, with a $4 \times 4 \times 4 k$ mesh and a plane-wave cutoff of $120 \mathrm{Ha}$.

The relaxed cubic lattice parameters obtained for $\mathrm{Si}$ and $\mathrm{SrTiO}_{3}$ are 10.102 and 7.267 bohrs, respectively. Calculations are performed under short-circuit electrostatic boundary conditions (see Refs. [10,12] for details). For $\mathrm{Si}$ and $\mathrm{SrTiO}_{3}, \mathrm{MP} k$ meshes from $4 \times 4 \times 4$ to $16 \times 16 \times 16$ and plane-wave cutoffs from 20 to $100 \mathrm{Ha}$ were tested to explore the convergence properties of the metric and phonon implementations. For the calculations of flexoelectric coefficients, a $12 \times 12 \times 12 k$ mesh and 80-Ha plane-wave cutoff were used.

\section{B. Charge-density response: Phonon vs metric}

First, we check the validity of Eq. (43), which connects the metric and phonon charge-density response functions via a geometric term. To make this test quantitative, we have taken advantage of the distance function defined in Eq. (54), with $f(\mathbf{r})=n_{\mathbf{q}}^{u^{\beta}}(\mathbf{r})$ and $g(\mathbf{r})=n_{\mathbf{q}}^{(\beta)}(\mathbf{r})+\Delta n_{\mathbf{q}}^{\beta}(\mathbf{r})$. [We construct $\Delta n_{\mathbf{q}}^{\beta}(\mathbf{r})$ in terms of the ground-state density, following Eq. (46).] Tests are conducted on $\mathrm{He}, \mathrm{Ne}$, and $\mathrm{Kr}$ atoms. Due to periodic boundary condition, these systems can be regarded as crystals of isolated atoms, intended as a computational analog to the toy model of Ref. [14], and discussed further in Sec. III E 1. The perturbations considered here are longitudinal, and they propagate along one of the three equivalent Cartesian axis. In Fig. 3 we report the values of $d(f, g)$ as a function of the wave-vector amplitude $|\mathbf{q}|$ for different energy cutoffs.

The first interesting observation is the almost perfect linear trend shown by the function $d(f, g)$. As we anticipated in Sec. II D, this is a direct consequence of using a finite planewave basis set: the larger the wave vector, the larger the shift of the cutoff sphere, and hence one expects a discrepancy that is roughly proportional to $|\mathbf{q}|$. 
Next, one can clearly appreciate, by comparing the slopes of the curves shown in Fig. 3, that the discrepancy between the phonon and metric results decreases as we increase the plane-wave cutoff. This happens because the discrepancy depends on the magnitude of the plane-wave coefficient at the boundary of the cutoff sphere (i.e., those falling outside the intersection of the two circles in Fig. 2); this is expected to decrease quickly with the cutoff, consistent with our results. Also, we see that the discrepancy between the metric and phonon charge-density responses is an order of magnitude less for $\mathrm{Kr}$ than $\mathrm{He}$ and $\mathrm{Ne}$; This is a direct consequence of the much softer pseudopotential associated to $\mathrm{Kr}$ as compared to $\mathrm{Ne}$ and $\mathrm{He}$.

As a final comment, we look at the calculated values corresponding to wave vectors $\mathbf{q}$ that are not necessarily commensurate with the $\mathbf{k}$ mesh. (For example, we have used an $8 \times 8 \times 8 \mathrm{MP} \mathbf{k}$ mesh for $\mathrm{He}$ and Ne systems, so the point $q=0.1$ does not match the $\mathbf{k}$ mesh.) The perfect linear trend of the distance function for all $\mathbf{q}$ values, irrespective of the exact or inexact cancellation in Eq. (45) (see discussion in Sec. II D), is a clear proof that the $\mathbf{k}$ mesh is dense enough, so that the finiteness of the plane-wave cutoff is the main source of error in this test.

We stress that the "discrepancies" that we discussed above are perfectly in line with the expected trends, and thus confirm the correctness of the implementation.

\section{Charge-density response: Metric vs uniform strain}

A second test of the metric implementation is based on its relationship with the response to a uniform strain. Indeed, the first derivative respect to the wave vector $\mathbf{q}$ of the metric perturbation should coincide with the strain perturbation of Hamann et al. [8], which is already implemented in the official release of the ABINIT code [see Eq. (31)]. To prove this point, here we use the distance function of Eq. (54) to compare the charge-density response functions $f(\mathbf{r})=-i n_{\alpha}^{1, \beta}(\mathbf{r})$ and $g(\mathbf{r})=n_{\alpha \beta}^{\text {strain }}(\mathbf{r})$, which should coincide according to Eq. (52). The derivative respect to $\mathbf{q}$ of the metric response is performed by finite differences, and using two different spacing values: $\Delta q=\left(2 \pi / a_{0}\right)\{0.01 ; 0.001\}$, where $a_{0}$ is the lattice parameter of the primitive cubic cell. In Fig. 4 we show $d(f, g)$ for the crystal of noninteracting $\mathrm{He}$ atoms as function of the energy cutoff $E_{\text {cut }}$. As expected, the discrepancy rapidly goes to zero at larger values of $E_{\text {cut }}$, again proving the correctness of the implementation. We also note that, by reducing the spacing value for the numerical calculation of the $\mathbf{q}$ derivative, the consistency between the metric and strain results increases by one order of magnitude.

\section{Octupoles}

We now compare the longitudinal octupoles calculated either using the metric or the standard acoustic phonon perturbation. Following the procedure described in Ref. [11], we interpolate the total density response of both the phonon and metric perturbations with a cubic polynomial as a function of q. For this test we have employed the three noble-gas atoms $(\mathrm{He}, \mathrm{Ne}$, and $\mathrm{Kr}$ ), $\mathrm{Si}$ in the diamond structure, and cubic $\mathrm{SrTiO}_{3}$. The directions chosen to calculate the longitudinal

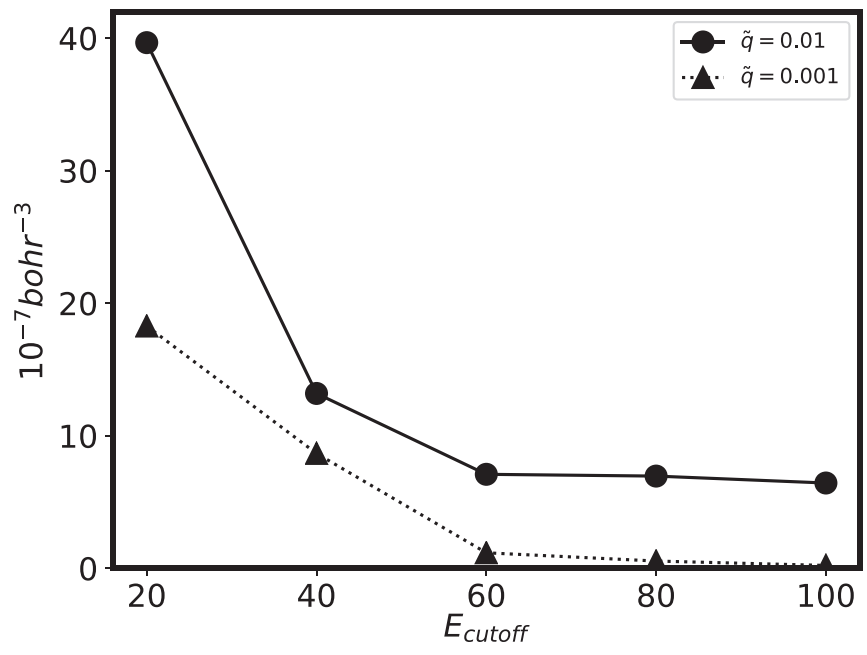

FIG. 4. Cell-integrated difference $d\left(-i n_{\alpha}^{1, \beta}(\mathbf{r}), n_{\alpha \beta}^{\text {strain }}(\mathbf{r})\right)$ between the first-order term of the long-wave expansion applied to the metric response density and the uniform strain response, calculated as in Ref. [8], for a He atom in a box undergoing a longitudinal mechanical perturbation. The two curves refer to two different values of the finite-difference increment used for obtaining $n_{\alpha}^{1, \beta}(\mathbf{r})$.

octupole are [100] and [110] for $\mathrm{Si}$ and $\mathrm{SrTiO}_{3}$, respectively, while the wave-vector amplitudes that we use for the cubic fit are $q_{i}=\{0.01 ; 0.02 ; 0.03\}$ (in reduced units of $2 \pi / a_{0}$ ). Note that, in the case of the phonon response, the electronic charge also has a nonzero linear term as a function of $\mathbf{q}$, whose slope gives the electronic contribution to the Born effective charge of the displaced sublattice. Such a linear term is not present in the metric response, as the atoms are not moving in the curvilinear frame.

In Table I we report the values of $Q_{L} / 6$ for the $\mathrm{He}, \mathrm{Ne}$, and $\mathrm{Kr}$ atoms, while the corresponding values for bulk $\mathrm{Si}$ and $\mathrm{SrTiO}_{3}$ are shown in Table II. As expected, the agreement between the metric and the phonon results increases with increasing the plane-wave cutoff; such an agreement becomes essentially perfect in the case of the isolated noble-gas atoms at an energy cutoff of $100 \mathrm{Ha}$. The metric results converge much faster as a function of $E_{\text {cut }}$ than the phonon results. Moreover, the test between the silicon octupole calculated with a $12 \times 12 \times 12$ and a $16 \times 16 \times 16$ MP k mesh shows that the metric calculation also converges much faster with respect to the number of $\mathbf{k}$ points.

The relatively worse convergence behavior in the phonon case can be tracked down to the quantity $\Delta n_{\mathbf{q}}^{\beta}(\mathbf{r})$. Indeed, if

TABLE I. $Q_{L} / 6$ for $\mathrm{He}$ and $\mathrm{Ne}$ atoms, in $e$ bohrs $^{2}$ (short-circuit electrostatic boundary conditions).

\begin{tabular}{|c|c|c|c|c|c|c|}
\hline \multirow{2}{*}{$\begin{array}{l}\text { Cutoff } \\
\text { (Ha) }\end{array}$} & \multicolumn{2}{|c|}{ He (5 bohrs) } & \multicolumn{2}{|c|}{$\mathrm{Ne}$ (7 bohrs) } & \multicolumn{2}{|c|}{$\mathrm{Kr}$ (14 bohrs) } \\
\hline & metr & phon & metr & phon & metr & phon \\
\hline 40 & 0.4392 & 0.4660 & 1.7338 & 1.7928 & 5.8433 & 5.8382 \\
\hline 60 & 0.4392 & 0.4322 & 1.8129 & 1.8208 & 5.8635 & 5.8618 \\
\hline 80 & 0.4396 & 0.4418 & 1.8135 & 1.8111 & 5.8635 & 5.8635 \\
\hline 100 & 0.4398 & 0.4398 & 1.8135 & 1.8135 & 5.8635 & 5.8635 \\
\hline
\end{tabular}


TABLE II. $Q_{L} / 6$ along the [100] direction for $\mathrm{Si}$ and along the [110] direction for $\mathrm{SrTiO}_{3}$. Values are in units of $e$ bohrs ${ }^{2}$ (short circuit (SC) electrostatic boundary conditions). The two different columns for Si refer to two different $\mathbf{k}$ meshes used: $12 \times 12 \times 12$ and $16 \times 16 \times 16$, respectively.

\begin{tabular}{|c|c|c|c|c|c|c|}
\hline \multirow{2}{*}{$\begin{array}{l}\text { Cutoff } \\
\text { (Ha) }\end{array}$} & \multicolumn{2}{|c|}{$\operatorname{Si}(12)$} & \multicolumn{2}{|c|}{$\operatorname{Si}(16)$} & \multicolumn{2}{|c|}{$\mathrm{STO}_{3}$} \\
\hline & metr & phon & metr & phon & metr & phon \\
\hline 20 & 478.379 & 478.456 & 478.391 & 478.409 & & \\
\hline 40 & 478.597 & 478.644 & 478.597 & 478.605 & & \\
\hline 60 & 478.601 & 478.653 & 478.601 & 478.612 & 111.793 & 111.658 \\
\hline 80 & 478.601 & 478.653 & & & 111.662 & 111.666 \\
\hline 100 & 478.601 & 478.653 & & & 111.684 & 111.673 \\
\hline
\end{tabular}

Eq. (46) were exactly satisfied, the cell integral of $\Delta n_{\mathbf{q}}^{\beta}(\mathbf{r})$ would vanish identically, and would not contribute to the calculated octupolar moment. However, as we have seen in Sec. II D, in practical calculations Eq. (46) is violated, and more so at lower-energy cutoffs or coarser k-point samplings. This can introduce an additional, spurious $\mathcal{O}\left(q^{3}\right)$ contribution to the macroscopic density response, and since $\Delta n_{\mathbf{q}}^{\beta}(\mathbf{r})$ is rather large, this can have a negative impact on the overall convergence. Thus, our numerical tests reveal a further (and formerly unexpected) advantage of the metric perturbation presented here, i.e., a significant economy in terms of computational resources compared with the standard phonon treatment. This can be important when dealing with larger systems; we shall come back to this point later on.

\section{E. Flexoelectric coefficients}

We will now perform calculations of FxE coefficients for our test cases, comparing the phonon implementation of Ref. [12] [Eqs. (7)-(9)] and the metric-wave method implemented in this work [Eqs. (10) and (11)]. We will report the flexoelectric tensor components in type-II form (types I and II are linearly related to one another), following the convention that was established in earlier works. Moreover, as we are dealing with cubic crystals, the flexoelectric tensor has only three independent components, which are indicated as the longitudinal $\left(\mu_{\mathrm{L}} \equiv \mu_{11,11}\right)$, transverse $\left(\mu_{\mathrm{T}} \equiv \mu_{11,22}\right)$, and shear $\left(\mu_{\mathrm{S}} \equiv \mu_{12,12}\right)$ flexoelectric coefficients henceforth (also remind that in cubic crystals the diamagnetic susceptibility is isotropic, $\chi_{\gamma \lambda}^{\mathrm{mag}}=\delta_{\gamma \lambda} \chi^{\mathrm{mag}}$ ). In all cases, the second derivatives with respect to $\mathbf{q}$ necessary for Eqs. (7) and (11) will be taken numerically with $\Delta q=\left(2 \pi / a_{0}\right) 0.005$.

\section{Isolated spherical atoms}

In order to test the metric implementation for calculating FxE coefficients, we consider the toy model of a material made of isolated (i.e., noninteracting), spherical charge densities that was explored in Refs. $[13,14,23]$. In earlier works, this was denoted as the isolated rigid charge (IRC) model, and we shall follow such naming convention here. Of course, such a material is fictitious since it would have no interatomic forces to hold it together. However, it serves as an interesting test case since its FxE properties can be determined analytically and compared to our numerical calculations. As before
TABLE III. Clamped-ion flexoelectric coefficients calculated using the metric and phonon implementations, as well as the quadrupole moments of the ground-state charge density. In the case of the phonon perturbation, the dynamic contribution, proportional to $\chi^{\mathrm{mag}}$, has been removed. All quantities are in units of $\mathrm{pC} / \mathrm{m}$.

\begin{tabular}{|c|c|c|c|c|c|c|c|}
\hline & \multicolumn{2}{|c|}{$\mu_{\mathrm{L}}^{\prime}$} & \multicolumn{2}{|c|}{$\mu_{\mathrm{T}}^{\prime}$} & \multirow[b]{2}{*}{$\epsilon Q_{\mathrm{NG}} / 2 \Omega$} & \multicolumn{2}{|c|}{$\mu_{\mathrm{S}}^{\prime}\left(10^{-3}\right)$} \\
\hline & metr & phon & metr & phon & & metr & phon \\
\hline $\mathrm{He}$ & -0.479 & -0.479 & -0.479 & -0.479 & -0.479 & 0.0 & -0.3 \\
\hline $\mathrm{Ne}$ & -1.843 & -1.844 & -1.841 & -1.842 & -1.842 & -0.7 & -0.6 \\
\hline $\mathrm{Kr}$ & -6.477 & -6.470 & -6.476 & -6.476 & -6.479 & -0.3 & -0.5 \\
\hline
\end{tabular}

[12], we can approximate this model by performing DFT calculations on noble-gas atoms in large enough simulation cells (filled with vacuum) that they do not interact with their periodic images. As pointed out in Ref. [12], however, in practical calculations atoms are not "rigid" but slightly polarizable, and the model needs to be revised to account for this fact.

Based on the revised IRC model, for the metric implementation (under short circuit boundary conditions) we expect that the flexoelectric coefficients will be $[12,13]$

$$
\mu_{\mathrm{NG}, \mathrm{L}}^{\prime}=\mu_{\mathrm{NG}, \mathrm{T}}^{\prime}=\epsilon \frac{Q_{\mathrm{NG}}}{2 \Omega}
$$

and

$$
\mu_{\mathrm{NG}, \mathrm{S}}^{\prime}=0,
$$

where the subscript "NG" indicates a DFT calculation on a noble-gas atom, $\epsilon$ is the isotropic clamped-ion dielectric constant, and $Q_{\mathrm{NG}}$ is the quadrupole moment of the groundstate charge density of the noble-gas atom. The presence of $\epsilon$ accounts for the fact that the noble-gas atoms (in contrast to the IRC model charge densities) are slightly polarizable [12], as we mentioned above.

Table III gives the clamped-ion FxE coefficients calculated for noble-gas atoms using the metric and phonon implementations. By comparing the $\mu_{\mathrm{L}}^{\prime}, \mu_{\mathrm{T}}^{\prime}$, and $\epsilon Q_{\mathrm{NG}} / 2 \Omega$ columns, we see that Eq. (55) is satisfied to within our numerical accuracy for both the metric and phonon methods. In addition, $\mu_{\mathrm{S}}^{\prime}$ vanishes (within our numerical accuracy). The main source of error is the numerical differentiation of the induced polarization with $\mathbf{q}$ in order to obtain Eqs. (7) and (11). The results of Table III indicate that the metric implementation is an accurate method for calculating flexoelectric coefficients, with increased efficiency as discussed above.

\section{Cubic materials}

We will now apply the metric implementation to calculate the bulk, clamped-ion FxE coefficients for two prototypical materials: $\mathrm{SrTiO}_{3}$ (in the high-temperature cubic phase) and $\mathrm{Si}$. As with the isolated atoms, we calculate the primed FxE coefficients from the phonon method by calculating $\chi^{\text {mag }}$ and using Eq. (9); the metric implementation gives us the prime coefficients directly. We can see from Table IV that the agreement between the metric and phonon implementations is excellent. 
TABLE IV. Flexoelectric constants for $\mathrm{SrTiO}_{3}$ and $\mathrm{Si}$ calculated using the phonon and metric implementations (units are $\mathrm{nC} / \mathrm{m}$ ); their orbital magnetic susceptibilities $\chi^{\mathrm{mag}}$ are, respectively, $-8.3 \times 10^{-3}$ and $10.2 \times 10^{-3} \mathrm{nC} / \mathrm{m}$.

\begin{tabular}{lccllllll}
\hline \hline & \multicolumn{2}{c}{$\mu_{\mathrm{L}}^{\prime}$} & & \multicolumn{2}{c}{$\mu_{\mathrm{T}}^{\prime}$} & & \multicolumn{2}{c}{$\mu_{\mathrm{S}}^{\prime}$} \\
\cline { 2 - 3 } \cline { 8 - 9 } \cline { 8 - 9 } & metr & phon & & metr & phon & & metr & phon \\
\hline $\mathrm{SrTiO}_{3}$ & -0.885 & -0.884 & & -0.826 & -0.826 & & -0.082 & -0.083 \\
$\mathrm{Si}$ & -1.411 & -1.410 & -1.049 & -1.050 & -0.189 & -0.190 \\
\hline \hline
\end{tabular}

As observed in previous calculations of the clamped-ion FxE coefficients [11,12], we see that $\mu_{\mathrm{L}}^{\prime} \simeq \mu_{\mathrm{T}}^{\prime} \gg \mu_{\mathrm{S}}^{\prime}$, which is similar to the behavior of the isolated atoms. However, a distinct difference is the importance of the dynamic contribution. We saw in Sec. III E 1 that for the isolated atoms, $\chi^{\text {mag }}$ was the same order as the longitudinal and transverse coefficients, whereas in the case of the cubic materials, the $\chi^{\mathrm{mag}}$ is two orders of magnitude smaller.

In spite of the small magnitude of $\chi^{\text {mag }}$, our results are sufficiently converged to see clearly that the rotation-gradient correction is required for accurate agreement between the metric and phonon implementations. If we neglect this correction, i.e., calculating $\mu$ with the phonon approach instead of $\mu^{\prime}$ [see Eq. (9)], we obtain $\mu_{\mathrm{T}}=-0.810$ and $\mu_{\mathrm{S}}=-0.091$ for $\mathrm{SrTiO}_{3}$, and $\mu_{\mathrm{T}}=-1.070$ and $\mu_{\mathrm{S}}=-0.180$ for $\mathrm{Si}$, which have clear discrepancies with the metric results in Table IV.

In Fig. 5 we show the convergence of the FxE coefficients of $\mathrm{SrTiO}_{3}$ and $\mathrm{Si}$ as a function of $k$-point mesh. We find that the metric implementation shows significantly more rapid convergence than the phonon implementation (they have similar convergence behavior with respect to plane-wave cutoff). The slower convergence of the phonon approach may have several possible origins. First, there may be additional numerical errors associated with the separate calculation of $\chi^{\text {mag }}$ [see Eq. (9)] that is needed for the phonon implementation but not for the metric. Also, as mentioned in Sec. II A 3, the expansion of the nonlocal contribution to the current density operator
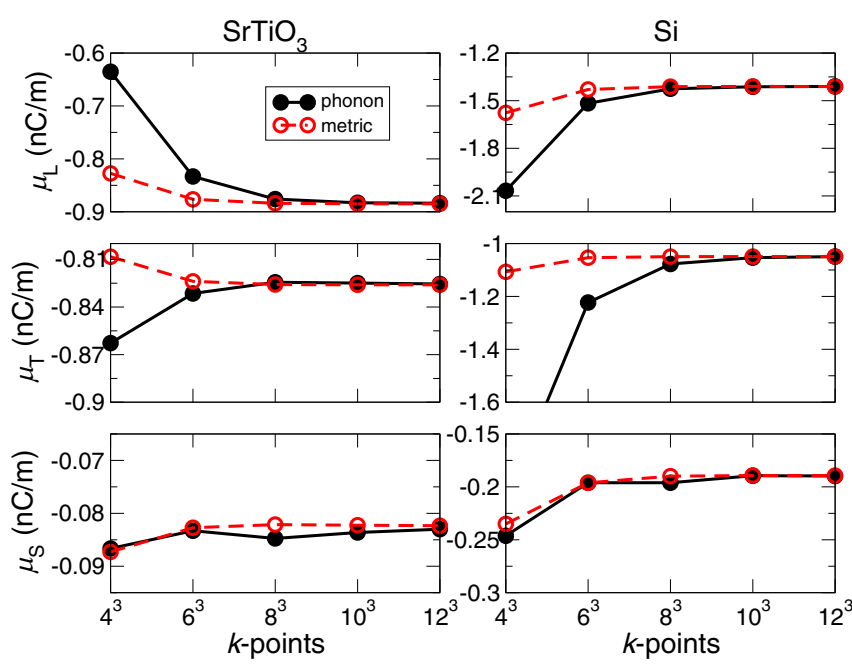

FIG. 5. Convergence of the FxE coefficients of $\mathrm{SrTiO}_{3}$ and $\mathrm{Si}$ with $k$-point mesh for the phonon and metric implementations. in the case of the metric implementation can be truncated to a lower order in $\mathbf{q}$ than in the phonon case. Finally, the two implementations differ with respect to the behavior of the local potential at $\mathbf{q}=0$, as will be discussed in Sec. III F.

These calculations clearly demonstrate the superiority of the metric implementation for determining the clamped-ion FxE coefficients. For the case of $\mathrm{SrTiO}_{3}$, for example, a calculation of the induced transverse polarization for a perturbation of a given $\mathbf{q}$ [i.e, Eqs. (8) and (10)] using the metric implementation took less than $17 \%$ of the CPU time of the phonon implementation, mostly because separate calculations for the different sublattices were not required. Additional savings in the calculation of the FxE coefficient also come from the fact that a calculation of $\chi^{\mathrm{mag}}$ is not required for the metric implementation.

\section{F. Discussion}

Here, we discuss a series of technical points related to the metric perturbation, together with possible future generalizations to other physical properties.

The first observation concerns the behavior of the "external potential" in the limit of $\mathbf{q} \rightarrow 0$. It is well known that the phonon perturbation diverges therein, and such divergence is carried by the $\mathbf{G}=0$ Fourier component of the local potential [sum over sublattices of Eq. (33)]

$$
\sum_{\kappa} V_{\mathbf{q}}^{\mathrm{loc}, \tau_{\kappa \beta}}(\mathbf{G}=0)=-i q_{\beta} \frac{1}{\Omega} \sum_{\kappa} v_{\kappa}^{\mathrm{loc}}(\mathbf{q}),
$$

where the contribution of each individual sublattice goes like

$$
v_{\kappa}^{\text {loc }}(\mathbf{q}) \sim 4 \pi \frac{Z_{\kappa}}{q^{2}} .
$$

(Recall that $Z_{\kappa}$ is the total pseudopotential charge.) The local pseudopotential contribution to the metric perturbation, Eq. (26), is characterized by an analogous divergence, but the latter is exactly canceled by an equal and opposite divergence in the geometric Hartree term (28):

$$
V_{\mathbf{q}}^{\mathrm{H} 0,(\beta)}(\mathbf{G}=0)=4 \pi i \frac{q_{\beta}}{q^{2}} n^{(0)}(\mathbf{G}=0)
$$

[recall that $n^{(0)}(\mathbf{G}=0)=(1 / \Omega) \sum_{\kappa} Z_{\kappa}$, as the cell must be overall charge neutral]. The fact that $\hat{H}_{\mathbf{k}, \mathbf{q}}^{(\beta)}$ remains finite (in fact, it vanishes) in the limit $\mathbf{q} \rightarrow 0$ might also help explain the superior numerical behavior of the metric perturbation in the convergence tests.

The second point we want to stress concerns the electrostatic boundary conditions used in this work. Throughout this work we have implicitly adopted short-circuit electrostatic boundary conditions (EBC) because we were mainly interested in long-wave expansions of the polarization (or charge-density) response; to do this, it is essential to deal with an analytic function, and the short-circuit EBC precisely remove the nonanalyticity generated by the presence of the macroscopic electric field [10]. This, however, differs from the physical electrostatic conditions ("mixed" EBC) that characterize the phonon response at nonzero $\mathbf{q}$ [9]. (The difference between the two cases only concerns the longitudinal deformations since with the mixed EBC the mechanical deformation generates a macroscopic electric field.) Thus, if the metric 
perturbation is to be employed for the realistic simulation of a finite-q acoustic phonon, such longitudinal fields must be incorporated in the calculation [11]. For a metric wave the short-circuit EBC are obtained by simply removing the $\mathbf{G}=$ 0 component from the self-consistent part of the first-order Hartree potential response $\hat{V}_{\mathbf{q}}^{(\beta)}$; by plugging this contribution back into the first-order Hamiltonian, we readily recover the correct electrostatics. Thus, switching from short-circuit to standard electrical boundary conditions is even simpler in the metric case than in the standard phonon case.

We conclude this section by briefly sketching other possible applications of the metric wave perturbation. In this work we have focused our attention on two response functions to the metric wave: the charge density [Eq. (46)] and the electronic polarization [Eq. (10)]. However, the knowledge of the wave functions $\left|u_{n \mathbf{k}, \mathbf{q}}^{(\beta)}\right\rangle$ can be used to calculate other useful physical quantities. An obvious candidate is the force-response tensor, defined by the force induced on the individual atomic sublattices by an acoustic phonon propagating along $\mathbf{q}$. This can be used [10] to calculate the lattice-mediated contributions to the flexoelectric tensor via an appropriate long-wave expansion in q. To see how, we can use a similar strategy as in the electronic polarization case, by exploiting the connection between the phonon and metric response functions. For example, we can write the "variational" contribution to the force experienced by the sublattice $\kappa$ in direction $\beta$ induced by an acoustic phonon as [21]

$$
f_{\kappa \beta, \alpha}^{(\mathrm{var}, \mathbf{q})}=\frac{4}{N_{\kappa}} \sum_{n \mathbf{k}}\left\langle u_{n \mathbf{k}, \mathbf{q}}^{u_{\alpha}}\left|H_{\mathbf{k}, \mathbf{q}}^{\tau_{\kappa \beta}}\right| u_{n \mathbf{k}}^{(0)}\right\rangle,
$$

where $\left|u_{n \mathbf{k}, \mathbf{q}}^{u_{\alpha}}\right\rangle=\sum_{\kappa^{\prime}}\left|u_{n \mathbf{k}, \mathbf{q}}^{\tau_{\kappa^{\prime}}}\right\rangle$ is the response to an acoustic phonon in the laboratory frame, defined as usual as the sublattice sum of individual atomic displacement. Then, $\left|u_{n \mathbf{k}, \mathbf{q}}^{u_{\alpha}}\right\rangle$ can be replaced, by using Eq. (40), with the metric response function $\left|u_{n \mathbf{k}, \mathbf{q}}^{(\alpha)}\right\rangle$, plus an additional piece that only depends on the ground-state wave functions, and can therefore be reabsorbed into the "nonvariational" part. Similar considerations could be used, in principle, to get the acoustic activity tensor [29] or the strain-gradient elastic tensor [30], which correspond to third and fourth orders in $\mathbf{q}$ of the metric-metric response.

\section{CONCLUSIONS}

In this work, we have implemented and tested a metric wave perturbation, defined as an acoustic phonon described in the frame that is comoving with the atoms, in the context of DFPT. It is aimed at calculating the physical response of a crystalline material to a generic mechanical deformation, and formally bridges the gap between the already available "phonon" [21] and "uniform strain" [8] perturbations. By focusing on the calculation of the flexoelectric tensor components, we have demonstrated, via extensive numerical validation, its clear advantages in terms of efficiency and ease of use with respect to earlier approaches. We also study its convergence properties with respect to various computational parameters, and find them to be very favorable. We rationalize this finding by comparing (both analytically and numerically) the charge-density response to the metric and standard phonon perturbations. We anticipate that, going forward, the method- ology presented here can become a standard approach for the first-principles computation of flexoelectric and related properties of materials.

\section{ACKNOWLEDGMENTS}

C.E.D. and D.V. were supported by ONR Grant No. N00014-16-1-2951. A.S. and M.S. acknowledge the support of Ministerio de Economia, Industria y Competitividad (MINECO-Spain) through Grants No. MAT2016-77100-C22-P and No. SEV-2015-0496, and of Generalitat de Catalunya (Grant No. 2017 SGR1506). This project has received funding from the European Research Council (ERC) under the European Union's Horizon 2020 research and innovation program (Grant Agreement No. 724529). Part of the calculations were performed at the Supercomputing Center of Galicia (CESGA). The Flatiron Institute is a division of the Simons Foundation.

\section{APPENDIX: DERIVATION OF THE PSEUDOPOTENTIAL TERMS}

Here, we carry out explicitly the derivation of the firstorder pseudopotential terms in curvilinear coordinates (26) and (27). The curvilinear coordinates are defined by Eq. (13). Following the results of Ref. [13], a generic (nonlocal) pseudopotential operator in the curvilinear coordinates is

$$
\tilde{V}^{\mathrm{psp},(0)}\left(\boldsymbol{\xi}, \boldsymbol{\xi}^{\prime}\right)=\sqrt{h(\xi)} V^{\mathrm{psp},(0)}\left(\mathbf{r}(\boldsymbol{\xi}), \mathbf{r}\left(\xi^{\prime}\right)\right) \sqrt{h\left(\boldsymbol{\xi}^{\prime}\right)},
$$

where $h(\xi)$ is the determinant of the deformation gradient tensor $h_{\alpha \beta}=\frac{\partial x_{\alpha}}{\partial \xi_{\beta}}$, that in the linear approximation is

$$
h(\boldsymbol{\xi})=1+i \mathbf{q} \cdot \lambda e^{i \xi \cdot \mathbf{q}} .
$$

\section{Local potential}

By using the transformation properties of the Dirac delta [see local term in Eq. (15)], one can easily verify that the factors of $\sqrt{h}$ cancel out in the local part

$$
\tilde{V}^{\mathrm{loc},(0)}(\boldsymbol{\xi})=V^{\mathrm{loc},(0)}(\mathbf{r}(\boldsymbol{\xi})) .
$$

Using Eqs. (16) and (13), this immediately leads to

$$
\begin{aligned}
\tilde{V}^{\mathrm{loc},(0)}(\boldsymbol{\xi})= & \sum_{l \kappa} v_{\kappa}^{\mathrm{loc}}\left[\boldsymbol{\xi}-\mathbf{R}_{l \kappa}+\lambda\left(e^{i \xi \cdot \mathbf{q}}-e^{i \mathbf{R}_{l \kappa} \cdot \mathbf{q}}\right)\right] \\
= & V^{\mathrm{loc},(0)}(\boldsymbol{\xi})+e^{i \xi \cdot \mathbf{q}} \sum_{l \kappa}\left[1-e^{i\left(\mathbf{R}_{l \kappa}-\xi\right) \cdot \mathbf{q}}\right] \\
& \times \lambda \cdot \nabla v_{\kappa}^{\text {loc }}\left(\boldsymbol{\xi}-\mathbf{R}_{l \kappa}\right)
\end{aligned}
$$

and, therefore, for the cell-periodic part of the first-order contribution

$$
V_{\mathbf{q}}^{\text {loc, }(\beta)}(\boldsymbol{\xi})=\sum_{l \kappa}\left[1-e^{i\left(\mathbf{R}_{l \kappa}-\xi\right) \cdot \mathbf{q}}\right] \frac{\partial}{\partial \xi_{\beta}} v_{\kappa}^{\text {loc }}\left(\boldsymbol{\xi}-\mathbf{R}_{l \kappa}\right) .
$$

Note the fact that the first-order potential vanishes identically at $\mathbf{q}=0$, which is a consequence of adopting the curvilinear reference system. 
To evaluate the Fourier transform, it is useful to bring the derivative sign out of the lattice sum in $V_{\mathbf{q}}^{\text {loc, }(\beta)}(\xi)$, obtaining the following three pieces:

$$
\begin{aligned}
V_{\mathbf{q}}^{\text {loc },(\beta)}(\boldsymbol{\xi})= & \frac{\partial}{\partial \xi_{\beta}} \sum_{l \kappa} v_{\kappa}^{\text {loc }}\left(\boldsymbol{\xi}-\mathbf{R}_{l \kappa}\right) \\
& -\frac{\partial}{\partial \xi_{\beta}}\left\{\sum_{l \kappa} e^{i \mathbf{q} \cdot\left(\mathbf{R}_{l \kappa}-\xi\right)} v_{\kappa}^{\text {loc }}\left(\boldsymbol{\xi}-\mathbf{R}_{l \kappa}\right)\right\} \\
& -i q_{\beta} \sum_{l \kappa} e^{i \mathbf{q} \cdot\left(\mathbf{R}_{l \kappa}-\xi\right)} v_{\kappa}^{\text {loc }}\left(\boldsymbol{\xi}-\mathbf{R}_{l \kappa}\right) .
\end{aligned}
$$

By defining [Ref. [21], Eq. (A16)] the Fourier transform of the local atomic potential,

$$
v_{\kappa}^{\text {loc }}(\mathbf{K})=\int d^{3} r e^{-i \mathbf{K} \cdot \mathbf{r}} v_{\kappa}^{\text {loc }}(\mathbf{r})
$$

we can readily evaluate the reciprocal-space expression for the perturbed local potential, which is precisely Eq. (26).

\section{Separable potential}

To evaluate the separable part, first recall that

$$
\begin{aligned}
\sqrt{h} & =1+\frac{i}{2} \lambda \cdot \mathbf{q} e^{i \xi \cdot \mathbf{q}}, \\
\zeta_{\mu \kappa}\left[\mathbf{r}(\boldsymbol{\xi})-\mathbf{r}\left(\mathbf{R}_{l \kappa}\right)\right]= & \zeta_{\mu \kappa}\left(\boldsymbol{\xi}-\mathbf{R}_{l \kappa}\right)+e^{i \xi \cdot \mathbf{q}}\left[1-e^{i\left(\mathbf{R}_{l k}-\boldsymbol{\xi}\right) \cdot \mathbf{q}}\right] \\
& \times \lambda \cdot \nabla \zeta_{\mu \kappa}\left(\boldsymbol{\xi}-\mathbf{R}_{l \kappa}\right) .
\end{aligned}
$$

It is also useful to remind some basic properties of the Fourier transformation of separable operators. Assume that we wish to express, in Fourier space, the following cell-periodic function:

$$
F\left(\mathbf{r}, \mathbf{r}^{\prime}\right)=\sum_{l} f\left(\mathbf{r}-\mathbf{R}_{l \kappa}\right) g^{*}\left(\mathbf{r}^{\prime}-\mathbf{R}_{l \kappa}\right) .
$$

We have, following Eq. (A19) of Ref. [21],

$$
F\left(\mathbf{G}+\mathbf{k}, \mathbf{G}^{\prime}+\mathbf{k}\right)=\frac{1}{\Omega} e^{i\left(\mathbf{G}^{\prime}-\mathbf{G}\right) \cdot \boldsymbol{\tau}_{k}} f(\mathbf{G}+\mathbf{k}) g^{*}\left(\mathbf{G}^{\prime}+\mathbf{k}\right) .
$$

Another basic relationship that we need is

$$
\int d^{3} r f^{*}(\mathbf{r}) e^{i \mathbf{K}^{\prime} \cdot \mathbf{r}}=\left(\int d^{3} r f(\mathbf{r}) e^{i \mathbf{K}^{\prime} \cdot \mathbf{r}}\right)^{*}=f^{*}\left(\mathbf{K}^{\prime}\right)
$$

We get, after some algebra,

$$
V_{\mathbf{k}, \mathbf{q}}^{\mathrm{sep},(\beta)}\left(\mathbf{G}, \mathbf{G}^{\prime}\right)=\frac{1}{\Omega} \sum_{\kappa \mu} e_{\mu \kappa} e^{i\left(\mathbf{G}^{\prime}-\mathbf{G}\right) \cdot \tau_{\kappa}} f_{\kappa \mu}^{(\beta, \mathbf{q})}\left(\mathbf{K}, \mathbf{K}^{\prime}\right),
$$

with (the first two terms come from the volume factors, third and fourth from the linear variation of $\zeta$, fifth and sixth from $\left.\zeta^{*}\right)$

$$
\begin{aligned}
& f_{\kappa \mu}^{(\beta, \mathbf{q})}\left(\mathbf{K}, \mathbf{K}^{\prime}\right) \\
&= \frac{i}{2} q_{\beta} \zeta(\mathbf{K}) \zeta^{*}\left(\mathbf{K}^{\prime}\right)+\frac{i}{2} q_{\beta} \zeta(\mathbf{K}+\mathbf{q}) \zeta^{*}\left(\mathbf{K}^{\prime}+\mathbf{q}\right) \\
&+i K_{\beta} \zeta(\mathbf{K}) \zeta^{*}\left(\mathbf{K}^{\prime}\right)-i\left(K_{\beta}+q_{\beta}\right) \zeta(\mathbf{K}+\mathbf{q}) \zeta^{*}\left(\mathbf{K}^{\prime}\right) \\
&-i\left(K_{\beta}^{\prime}+q_{\beta}\right) \zeta(\mathbf{K}+\mathbf{q}) \zeta^{*}\left(\mathbf{K}^{\prime}+\mathbf{q}\right) \\
&+i K_{\beta}^{\prime} \zeta(\mathbf{K}+\mathbf{q}) \zeta^{*}\left(\mathbf{K}^{\prime}\right) .
\end{aligned}
$$

This expression can be further simplified as follows:

$$
\begin{aligned}
f_{\kappa \mu}^{(\beta, \mathbf{q})}\left(\mathbf{K}, \mathbf{K}^{\prime}\right)= & i\left(K_{\beta}+\frac{q_{\beta}}{2}\right) \zeta(\mathbf{K}) \zeta^{*}\left(\mathbf{K}^{\prime}\right) \\
& -i\left(K_{\beta}^{\prime}+\frac{q_{\beta}}{2}\right) \zeta(\mathbf{K}+\mathbf{q}) \zeta^{*}\left(\mathbf{K}^{\prime}+\mathbf{q}\right) \\
& -i\left(K_{\beta}-K_{\beta}^{\prime}+q_{\beta}\right) \zeta(\mathbf{K}+\mathbf{q}) \zeta^{*}\left(\mathbf{K}^{\prime}\right)
\end{aligned}
$$

The final result is precisely Eq. (27).
[1] P. Zubko, G. Catalan, A. Buckley, P. R. L. Welche, and J. F. Scott, Phys. Rev. Lett. 99, 167601 (2007).

[2] M. S. Majdoub, P. Sharma, and T. Cagin, Phys. Rev. B 77, 125424 (2008).

[3] J. Narvaez, F. Vasquez-Sancho, and G. Catalan, Nature (London) 538, 219 (2016).

[4] U. K. Bhaskar, N. Banerjee, A. Abdollahi, Z. Wang, D. G. Schlom, G. Rijnders, and G. Catalan, Nat. Nanotechnol. 11, 263 (2016).

[5] H. Lu, C.-W. Bark, D. Esque de los Ojos, J. Alcala, C. B. Eom, G. Catalan, and A. Gruverman, Science 336, 59 (2012).

[6] L. Cross, J. Mater. Sci. 41, 53 (2006).

[7] R. M. Martin, Phys. Rev. B 5, 1607 (1972).

[8] D. R. Hamann, X. Wu, K. M. Rabe, and D. Vanderbilt, Phys. Rev. B 71, 035117 (2005).

[9] J. Hong and D. Vanderbilt, Phys. Rev. B 88, 174107 (2013).

[10] M. Stengel, Phys. Rev. B 88, 174106 (2013).

[11] M. Stengel, Phys. Rev. B 90, 201112 (2014).
[12] C. E. Dreyer, M. Stengel, and D. Vanderbilt, Phys. Rev. B 98, 075153 (2018).

[13] M. Stengel and D. Vanderbilt, Phys. Rev. B 98, 125133 (2018).

[14] M. Stengel, Nat. Commun. 4, 2693 (2013).

[15] A. K. Tagantsev, Phys. Rev. B 34, 5883 (1986).

[16] J. Sakuri and J. Napolitano, Modern Quantum Mechanics, 2nd ed. (Addison-Wesley, San Francisco, 1994).

[17] A. K. Tagantsev and A. S. Yurkov, J. Appl. Phys. 112, 044103 (2012).

[18] L. Kleinman and D. M. Bylander, Phys. Rev. Lett. 48, 1425 (1982).

[19] S. Ismail-Beigi, E. K. Chang, and S. G. Louie, Phys. Rev. Lett. 87, 087402 (2001).

[20] A. M. Essin, A. M. Turner, J. E. Moore, and D. Vanderbilt, Phys. Rev. B 81, 205104 (2010).

[21] X. Gonze, Phys. Rev. B 55, 10337 (1997).

[22] S. Baroni, S. de Gironcoli, A. Dal Corso, and P. Giannozzi, Rev. Mod. Phys. 73, 515 (2001). 
[23] M. Stengel and D. Vanderbilt, in Flexoelectricity in Solids From Theory to Applications, edited by A. K. Tagantsev and P. V. Yudin (World Scientific, Singapore, 2016), Chap. 2, pp. 31-110.

[24] X. Gonze and C. Lee, Phys. Rev. B 55, 10355 (1997).

[25] J. P. Perdew and Y. Wang, Phys. Rev. B 45, 13244 (1992).
[26] N. Troullier and J. L. Martins, Phys. Rev. B 43, 1993 (1991).

[27] M. Fuchs and M. Scheffler, Comput. Phys. Commun. 119, 67 (1999).

[28] H. J. Monkhorst and J. D. Pack, Phys. Rev. B 13, 5188 (1976).

[29] D. L. Portigal and E. Burstein, Phys. Rev. 170, 673 (1968).

[30] M. Stengel, Phys. Rev. B 93, 245107 (2016). 